\title{
TASI Lectures on Large $N$ Tensor Models
}

\section{Igor R. Klebanov*}

Department of Physics, Princeton University, Princeton, NJ 08544, USA

E-mail: klebanoveprinceton.edu

\section{Fedor Popov}

Department of Physics, Princeton University, Princeton, NJ 08544, USA

E-mail: fpopov@princeton.edu

\section{Grigory Tarnopolsky}

Department of Physics, Harvard University, Cambridge, MA 02138, USA

E-mail: grtarnop@g.harvard.edu

\begin{abstract}
The first part of these lecture notes is mostly devoted to a comparative discussion of the three basic large $N$ limits, which apply to fields which are vectors, matrices, or tensors of rank three and higher. After a brief review of some physical applications of large $N$ limits, we present a few solvable examples in zero space-time dimension. Using models with fields in the fundamental representation of $O(N), O(N)^{2}$, or $O(N)^{3}$ symmetry, we compare their combinatorial properties and highlight a competition between the snail and melon diagrams. We exhibit the different methods used for solving the vector, matrix, and tensor large $N$ limits. In the latter example we review how the dominance of melonic diagrams follows when a special "tetrahedral" interaction is introduced. The second part of the lectures is mostly about the fermionic quantum mechanical tensor models, whose large $N$ limits are similar to that in the Sachdev-Ye-Kitaev (SYK) model. The minimal Majorana model with $O(N)^{3}$ symmetry and the tetrahedral Hamiltonian is reviewed in some detail; it is the closest tensor counterpart of the SYK model. Also reviewed are generalizations to complex fermionic tensors, including a model with $S U(N)^{2} \times O(N) \times U(1)$ symmetry, which is a tensor counterpart of the complex SYK model. The bosonic large $N$ tensor models, which are formally tractable in continuous spacetime dimension, are reviewed briefly at the end.
\end{abstract}

Theoretical Advanced Study Institute Summer School 2017 "Physics at the Fundamental Frontier" 4 June - 1 July 2017

Boulder, Colorado

${ }^{*}$ Speaker. 


\section{Introduction and Summary}

An important tool in theoretical physics is the study of limits where the number of degrees of freedom, $N_{\text {dof }}$, becomes large. Some models simplify or even become analytically solvable in such a limit. If an (asymptotic) expansion in inverse powers of $N_{\text {dof }}$ can be developed, then models of physical interest are sometimes well approximated by including a small number of terms. Three different broad classes of such "large $N$ limits" have been explored: the vector limit; the matrix (or 't Hooft) limit; and, more recently, the limit which applies to tensors of rank three and higher. Each of them is addressed with somewhat different techniques.

The most easily tractable, and historically the first, large $N$ limit applies to theories where the degrees of freedom transform as $N$-component vectors under a symmetry group. In such theories $N_{\text {dof }} \sim N$. One of the first appearances was in the context of statistical mechanics [1]. A wellknown example is the $O(N)$ symmetric quantum theory of $N$ scalar fields $\phi^{a}$ in $d$ dimensions with interaction $\frac{g}{4}\left(\phi^{a} \phi^{a}\right)^{2}$ (for reviews see [2,3]). It is exactly solvable in the large $N$ limit where $g N$ is held fixed, since summation over the necessary class of bubble diagrams is not hard to evaluate. The $1 / N$ expansion may be developed analytically for any space-time dimension $d$.

Another famous large $N$ limit occurs in models of interacting $N \times N$ matrix fields, so that $N_{\text {dof }} \sim N^{2}$. This limit made its first appearance in the context of generalizing QCD from $S U(3)$ to $S U(N)$ gauge theory, where the gauge fields are traceless Hermitian $N \times N$ matrices [4]. A significant simplification occurs in the 't Hooft large $N$ limit where $g_{\mathrm{YM}}^{2} N$ is held fixed: the perturbative expansion is dominated by the planar diagrams. ${ }^{1}$ While the 't Hooft large $N$ limit does not make QCD in dimension above 2 exactly solvable, it has been an important tool in studying its properties. Furthermore, the 't Hooft large $N$ limit was crucial for the discovery and exploration of the Anti-de Sitter/Conformal Field Theory (AdS/CFT) correspondence [6-8], which has been a major research direction for over 20 years. For introductions to the AdS/CFT correspondence you may consult the lectures by O. DeWolfe [9], J. Erdmenger [10], and D. Harlow [11] in this volume, lectures at the earlier TASI schools including [12-14], and the comprehensive review [15].

Besides the gauge theories, the 't Hooft large $N$ limit applies to matrix models, such as the integral over of a Hermitian matrix $\Phi$ with single-trace interactions like $g_{3} \operatorname{tr} \Phi^{3}$ (see section 3.4). Such matrix models are exactly solvable in the large $N$ limit keeping $g_{3}^{2} N$ fixed, in the special lowdimensional cases $d \leq 1$ [16]. Here the planar graphs may be thought of as discretized random surfaces. Tuning $g_{3}^{2} N$ to a special value where a random surface becomes macroscopic [17-19] (for reviews see [20,21]) has taught us a lot about the two-dimensional quantum gravity, which can be mapped to the quantum Liouville theory [22].

In view of these classic results, it is natural to study theories with rank- $m$ tensor degrees of freedom $\phi^{a_{1} \ldots a_{m}}$, where each index takes $N$ values so that $N_{\text {dof }} \sim N^{m}$ [23-25]. Since the complexity of taking the large $N$ limit increases from $m=1$ to $m=2$, one might expect that the tensor models with $m>2$ are much more difficult than the matrix models. However, by choosing the interactions appropriately, it is possible to find models with $m>2$ where a large $N$ limit is solvable [26-35] (for

\footnotetext{
${ }^{1}$ This is true if the number of flavors $N_{f}$, i.e. fields in the fundamental representation of $S U(N)$, is held fixed in the 't Hooft limit. However, if $x=N_{f} / N$ is held fixed in the large $N$ limit, then the leading terms depend on the parameter $x$. This is known as the Veneziano limit [5]. This type of limit can be applied to any theory containing both matrix and vector fields.
} 
reviews, see [36-38]). The perturbative expansion is then dominated by special classes of Feynman diagrams. While the original hope for applications of these tensor models lay with quantum gravity above two dimensions, starting in October 2016 a new physical connection was opened up [33,34] with the Sachdev-Ye-Kitaev (SYK) model [39-43] (for recent reviews of the SYK model, see $[44,45])$. The $q=4$ version of the SYK model involves a large number $N_{\mathrm{SYK}}$ of Majorana fermions $\psi^{i}$, interacting via the quartic Hamiltonian

$$
H_{\mathrm{SYK}}=\sum_{i_{1}<i_{2}<i_{3}<i_{4}} J_{i_{1} i_{2} i_{3} i_{4}} \psi^{i_{1}} \psi^{i_{2}} \psi^{i_{3}} \psi^{i_{4}},
$$

where each $J_{i_{1} i_{2} i_{3} i_{4}}$ is a Gaussian random variable with standard deviation $\sim N_{\mathrm{SYK}}^{-3 / 2}$. The closest tensor counterpart [34] of this model contains $N^{3}$ Majorana fermions $\psi^{a b c}, a, b, c=1, \ldots, N$,

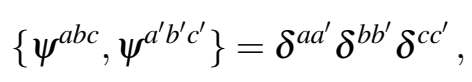

whose interactions are governed by the $O(N)^{3}$ symmetric "tetrahedral" Hamiltonian

$$
H=\frac{g}{4}\left(\psi^{a b c} \psi^{a b^{\prime} c^{\prime}} \psi^{a^{\prime} b c^{\prime}} \psi^{a^{\prime} b^{\prime} c}-\frac{1}{4} N^{4}\right)
$$

In this case the large $N$ limit has to be taken keeping $g^{2} N^{3}$ fixed. Then the diagrammatic expansion is dominated by the so-called melonic diagrams (see figure 1); they are obtained by iterating the melon (or sunset) propagator insertion shown on the right in figure 2.
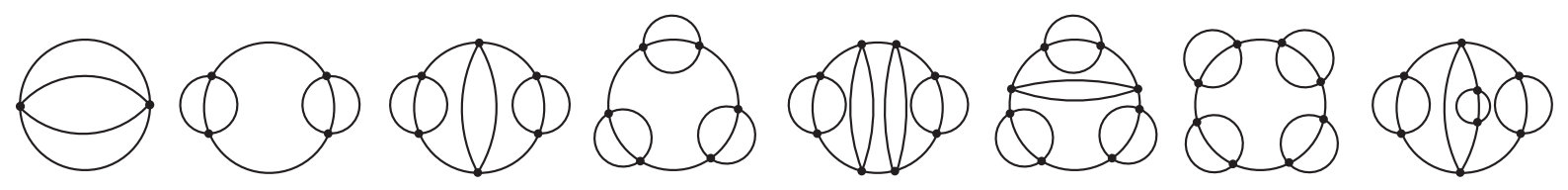

Figure 1: All the melonic vacuum diagrams up to order $g^{8}$.

Besides the three "basic" large $N$ limits mentioned above, there are more exotic examples where $N_{\text {dof }}$ can scale as a fractional power of $N$ in the large $N$ limit. Such limits are possible in presence of additional symmetries, such as extended supersymmetry. For example, in the context of the ABJM model [46], which is the $U(N)_{k} \times U(N)_{-k}$ Chern-Simons gauge theory in $d=3$ coupled to massless matter, it is possible to take the large $N$ limit while keeping $k$ fixed (this is is different from the 't Hooft limit where $N / k$ is held fixed). In this so-called "M-theory limit", there is strong evidence that the ABJM theory is dual to M-theory on the $A d S_{4} \times S^{7} / Z_{k}$ background, and one finds $N_{\text {dof }} \sim k^{1 / 2} N^{3 / 2}$ [47-50] (for reviews see [51,52]). An even more exotic situation is when the Chern-Simons theory is $U(N)_{k_{1}} \times U(N)_{k_{2}}$ with $k_{1}+k_{2} \neq 0$; then $N_{\text {dof }} \sim\left|k_{1}+k_{2}\right|^{1 / 3} N^{5 / 3}[53]$. Such "exotic" large $N$ limits are fascinating, but we will not discuss them further in these lectures.

In section 2 we review some classic applications of the vector and matrix large $N$ limits, including the $O(N)$ magnets and $S U(N)$ gauge theories. We also discuss briefly the planar $\mathscr{N}=4$ supersymmetric Yang-Mills theory in $d=4$ and the AdS/CFT correspondence. Section 3 contains a few solvable large $N$ examples in $d=0$. Using integrals over bosonic variables in the fundamental representation of $O(N), O(N)^{2}$, or $O(N)^{3}$ symmetry, we compare the different combinatorial properties of the three basic large $N$ limits. We highlight a competition between the snail and melon diagrams illustrated in figure 2. In section 4 we explain, following [32,34], why the melonic diagrams 

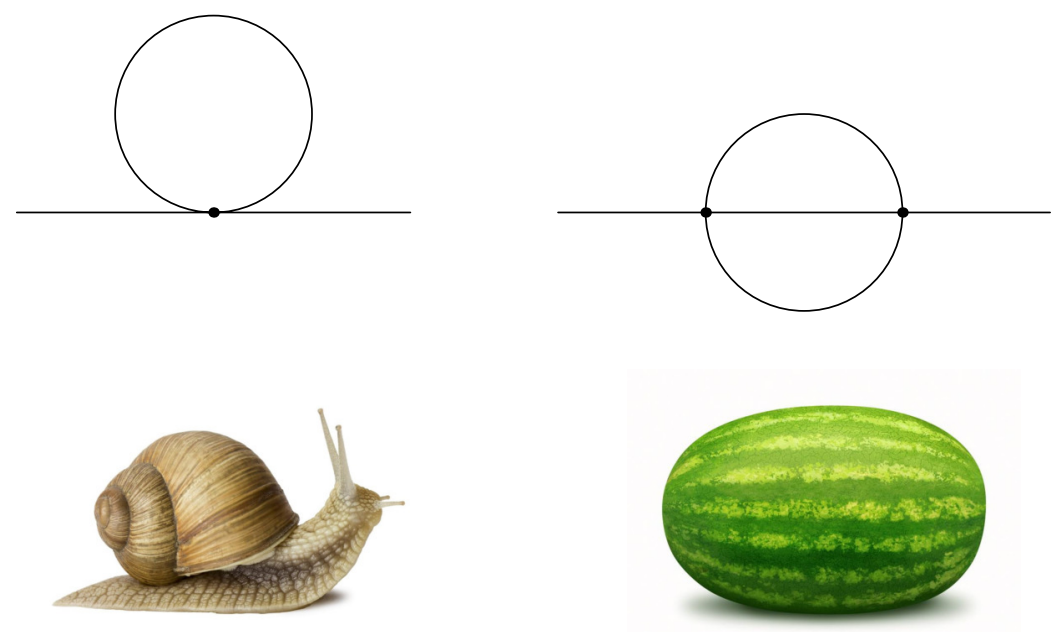

Figure 2: A snail vs. a (water)melon.

are dominant in large $N$ theories with $O(N)^{3}$ symmetry and the tetrahedral quartic interaction. In section 5 we review in some detail that $O(N)^{3}$ symmetric Majorana quantum mechanics (1.3) and its comparison with the SYK model (1.1). We explain why the $O(N)^{3}$ tensor model Hamiltonian is much more sparse than in the SYK model with $N^{3}$ fermions. Nevertheless, some quantities in the two models have the same large $N$ behavior, which can be shown using the Schwinger-Dyson equations. A novel feature of the tensor model is that the number of $S O(N)^{3}$ invariant $2 k$-particle operators grows as $k ! 2^{k}$ [54]; as a result, the Hagedorn temperature vanishes in the large $N$ limit. In section 6 we review the complex fermionic tensor model with $S U(N)^{2} \times O(N) \times U(1)$ symmetry [34], which is a tensor counterpart of the complex SYK model $[55,56]$. We also present new results on a complex bipartite model with $O(N)^{3}$ symmetry; its spectrum contains an operator with a complex scaling dimension. Such complex dimensions of the form $\frac{d}{2}+i \alpha(d)$ also appear in some large $N$ bosonic tensor models, which are briefly reviewed in section 7 . However, in the $O(N)^{3}$ model with the sixth-order "prismatic" interaction [35], there are ranges of $d$ where the large $N$ theory appears to be free of the complex scaling dimensions.

These notes are far from a comprehensive review, but we hope that they will give the reader a sense of the variety of large $N$ models that have been studied over many years. It is clear that their exploration is far from over. There may well be even more sophisticated large $N$ limits that are yet to be discovered, and interesting quantum theories which realize them.

\section{Some Applications of the Vector and Matrix Large $N$ Limits}

A classic application of the vector large $N$ limit is to the $O(N)$ ferromagnet, which is described by the following energy with $J>0$ (see figure 3)

$$
\mathscr{E}=-J \sum_{\langle i j\rangle} \vec{n}_{i} \cdot \vec{n}_{j}, \quad \vec{n}=\left(n^{1}, \ldots, n^{N}\right), \quad \vec{n}^{2}=1,
$$


where $\langle i j\rangle$ denotes the nearest neighbor lattice sites. The partition function is

$$
Z=\sum_{\left\{\vec{n}_{i}\right\}} e^{-\beta \mathscr{E}},
$$

where the sum is over all possible choices of $\vec{n}$ vectors at the lattice sites. This model has a second

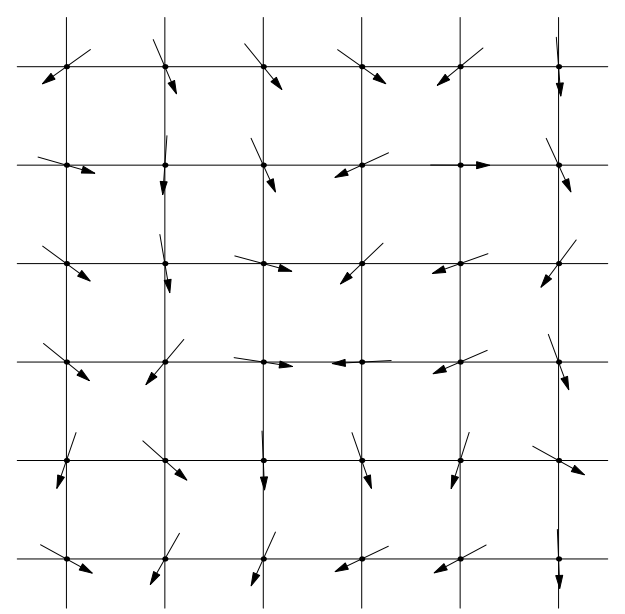

Figure 3: A configuration of spins in an $O(2)$ model on a two-dimensional lattice.

order phase transition at a critical value of $\beta J$, near which it is described by the Euclidean field theory, as reviewed in $[2,3]$ :

$$
S=\int d^{d} x\left[\frac{1}{2}\left(\nabla \phi_{i}\right)^{2}+\frac{m^{2}}{2} \phi_{i}^{2}+\frac{g}{4}\left(\phi_{i}^{2}\right)^{2}\right] .
$$

The phase transition separates the phases with $m^{2}>0$ and $m^{2}<0$. For $N>2$ the second-order transition is present for $d>2$, while for $N=1,2$ it is present also for $d=2$. In general the field theory (2.3) is super-renormalizable in $d<4$, and for $2<d<4$ it flows to the interacting infrared fixed point. In $d=4-\varepsilon$ the IR stable zero of the beta function is at weak coupling:

$$
\beta_{g}=-\varepsilon g+\frac{N+8}{8 \pi^{2}} g^{2}+O\left(g^{3}\right) \Rightarrow g_{*}=\frac{8 \pi^{2}}{N+8} \varepsilon+O\left(\varepsilon^{2}\right) .
$$

Substituing $g_{*}$ into scaling dimension of various operators produces their $\varepsilon$ expansion. The field $\phi_{i}$ is the spin operator, and $\phi_{i}^{2}$ is the "energy" operator - it is the simplest $O(N)$ invariant "single-trace" operator. Their scaling dimensions have $\varepsilon$ expansions

$$
\Delta_{\phi_{i}}=1-\frac{1}{2} \varepsilon+\frac{N+2}{4(N+8)^{2}} \varepsilon^{2}+O\left(\varepsilon^{3}\right), \quad \Delta_{\phi^{2}}=2-\frac{6}{N+8} \varepsilon+O\left(\varepsilon^{2}\right) .
$$

Extending them to higher orders in $\varepsilon$ and extrapolating to $\varepsilon=1$ gives accurate estimates of the scaling dimensions in the $d=3$ critical $O(N)$ model for all values of $N$, including $N=1$ which corresponds to the Ising model.

Another tool for studying the critical $O(N)$ model is the $1 / N$ expansion. It may be developed for the IR fixed point in $2<d<4$ by introducing the auxiliary field $\sigma$ (for a review, see the TASI 2015 lectures [57]):

$$
S_{\sigma}=\int d^{d} x\left[\frac{1}{2}\left(\nabla \phi_{i}\right)^{2}-\frac{i}{2} \sigma \phi_{i}^{2}+\frac{1}{4 g} \sigma^{2}\right]
$$


After integrating out the $N$ fields $\phi^{i}$, the field $\sigma$ acquires an induced effective action of order $N$, which dominates in the IR over the $\sigma^{2}$ term. Using the induced $\sigma$ propagator, which is of order $1 / N$, leads to the $1 / N$ expansions for the scaling dimensions. While they are available as functions of $d[3,58]$, we will just state them for the physically interesting case $d=3$ :

$$
\Delta_{\phi_{i}}=\frac{1}{2}+\frac{4}{3 \pi^{2} N}-\frac{256}{27 \pi^{4} N^{2}}+\ldots, \quad \Delta_{\phi^{2}}=2-\frac{32}{3 \pi^{2} N}+\frac{32\left(16-27 \pi^{2}\right)}{27 \pi^{4} N^{2}}+\ldots
$$

Precise results [59] from applications of the numerical conformal bootstrap [60] (for a recent review, see [61]) to the $d=3$ critical $O(N)$ model show an excellent match with these $1 / N$ expansions for $N>10$.

The $d=3$ critical $O(N)$ model has another interesting application - to higher spin quantum gravity. It has been conjectured [62] that its $O(N)$ singlet sector is dual to the minimal Vasiliev higher-spin theory in $A d S_{4}$ [63]. This conjecture and its generalizations have passed a number of non-trivial tests; for reviews see [57,64].

Since its introduction in 1974 [4], the 't Hooft large $N$ limit has had a multitude of applications. The recent ones include lattice calculations of bound state masses in $S U(N)$ gauge theory for moderate values of $N$, and their large $N$ extrapolations. For example, in the 3-dimensional pure glue $S U(N)$ theory, where the numerical results are particularly accurate, it was found [65] that the masses of low-lying glueballs exhibit a very good fit with the expansions

$$
\frac{m}{g_{\mathrm{YM}}^{2} N}=a_{0}+\frac{a_{1}}{N^{2}}+\frac{a_{2}}{N^{4}}+\ldots
$$

for values of $N$ in ranging from 2 to 16 . This constitutes a nice non-perturbative check of the large $N$ expansion which appears in the 't Hooft limit.

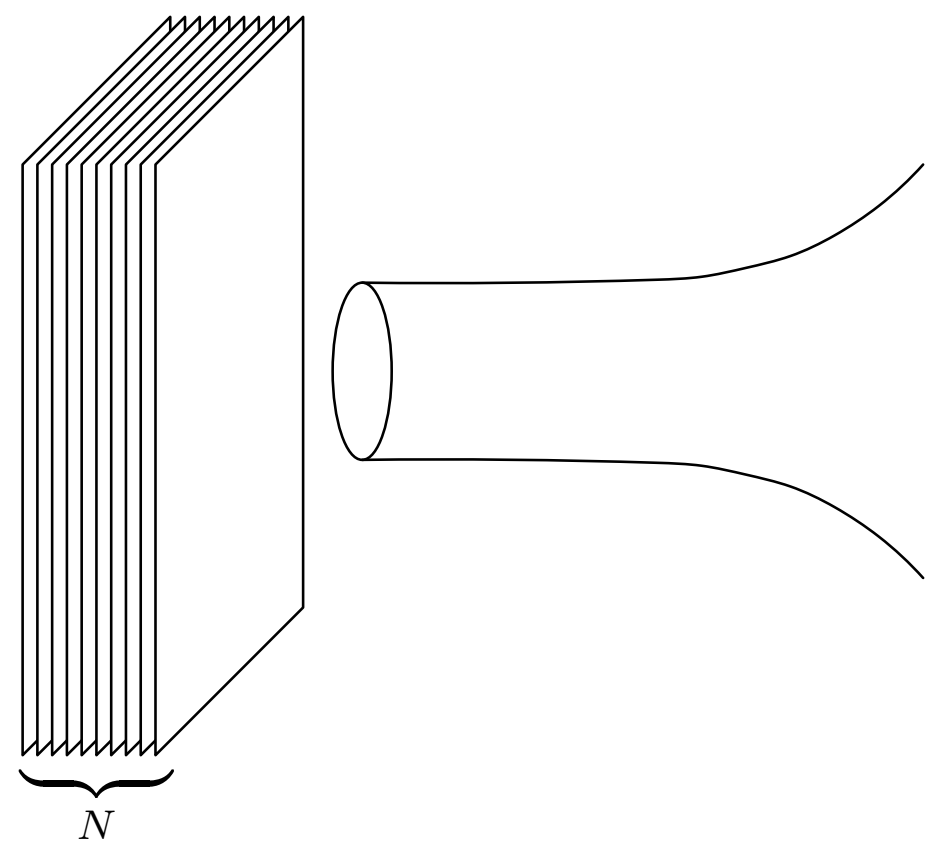

Figure 4: A stack of $N$ D3-branes and the curved background it creates. 
The 't Hooft large $N$ limit also plays an important role in the correspondence [6-8] between the $\mathscr{N}=4$ supersymmetric $S U(N)$ gauge in $d=4$ and the type IIB string theory on the $A d S_{5} \times S^{5}$ background. To arrive at this correspondence, it is convenient $[6,66]$ to begin with a stack of a large number $N$ of Dirichlet 3-branes (for an original review of D-branes, see the TASI 1996 lectures [67]). At low energies, this stack has two equivalent descriptions illustrated in figure 4. The first involves the $\mathscr{N}=4$ supersymmetric $S U(N)$ gauge theory weakly coupled to the type IIB closed superstrings. Since D-branes carry Ramond-Ramond charges [68], the second description involves type IIB closed superstring theory in the background of the extreme Ramond-Ramond charged 3-brane, which has the metric

$$
d s^{2}=h^{-\frac{1}{2}}(r)\left(-d t^{2}+d x_{i}^{2}\right)+h^{\frac{1}{2}}(r)\left(d r^{2}+r^{2} d \Omega_{5}^{2}\right), \quad h(r)=1+\frac{L^{4}}{r^{4}},
$$

where $d \Omega_{5}^{2}$ is the metric of a round unit 5-sphere. Equating the ADM tension of this extreme gravitational background to $N$ times the $\mathrm{D} 3$-brane tension gives

$$
L^{4}=\lambda \alpha^{\prime 2}, \quad \lambda \equiv g_{\mathrm{YM}}^{2} N,
$$

which implies that the curvature is small everywhere in string units when the 't Hooft coupling $\lambda$ is large. The low-energy limit may be taken directly in the geometry by sending $r \rightarrow 0$ [6], which corresponds to omitting the first term 1 in $h(r)$. The resulting metric

$$
d s^{2} \approx \frac{r^{2}}{L^{2}}\left(-d t^{2}+d x_{i}^{2}\right)+L^{2} \frac{d r^{2}}{r^{2}}+L^{2} d \Omega_{5}^{2}
$$

is that of a product of (the Poincare patch of) the 5-dimensional negatively curved anti-de Sitter space, $A d S_{5}$, and the 5-dimensional positively curved sphere, $S^{5}$. The two 5-dimensional spaces have equal curvature radii $L$.

The dimensionless parameter which measures the effects of quantum gravity, i.e. the string loop corrections in $A d S_{5} \times S^{5}$, is $G_{10} / L^{8}$, where $G_{10}$ is the ten-dimensional Newton constant. This is found to be of order $1 / N^{2}$. Therefore, the 't Hooft large $N$ limit corresponds to the classical limit of string theory. While the AdS/CFT correspondence has been conjectured to be valid at any value of $\lambda$, a crucial additional simplification occurs when it is taken to be very large. In this regime, the string theory may be well-approximated by the effective supergravity, and the effect of higherderivative stringy currections to the effective action is suppressed. This provides a remarkable application of the methods of Einstein's theory of gravity - this time to strongly coupled large $N$ QFT!

The AdS/CFT dictionary $[7,8]$ includes the one-to-one correspondence between gauge invariant scalar operators of scaling dimension $\Delta$ and scalar fields of mass-squared

$$
m^{2}=\frac{\Delta(\Delta-4)}{L^{2}}
$$

For example, we can consider the chiral primary operators

$$
O_{k}=\operatorname{tr} \Phi^{\left(i_{1}\right.} \Phi^{i_{2}} \ldots \Phi^{\left.i_{k}\right)},
$$

which are symmetric traceless polynomials made of the six scalar fields $\Phi_{i}$ contained in the $\mathscr{N}=4$ SYM theory; the scalars are in the adjoint representation of the gauge group and in the 6 of the 
R-symmetry group $S U(4) \sim S O(6)$. These operators are protected by supersymmetry and have exact dimension $\Delta=k$ (this can be checked perturbatively at small $\lambda$ ). On the AdS side they are dual to the Kaluza-Klein modes on the $S^{5}$ which indeed have $m^{2} L^{2}=k(k-4), k=2,3, \ldots$ [69]. This provided one of the first tests of the AdS/CFT correspondence.

The planar $\mathscr{N}=4$ supersymmetric Yang-Mills theory has another remarkable property, the exact integrability [70] (for a comprehensive review see [71]). The integrability has allowed matching of the perturbative expansions of some quantities, evaluated using the planar diagrams for $\lambda \ll 1$, to the predictions of string theory in weakly curved $A d S_{5} \times S^{5}$ valid for $\lambda \gg 1$. Consider, for example, another operator made out of the adjoint scalars: an $S O(6)$ singlet known as the Konishi operator,

$$
O_{\text {Konishi }}=\sum_{i=1}^{6} \operatorname{tr} \Phi_{i}^{2}
$$

Its dimension is not protected by supersymmetry, as can be seen from the perturbative planar expansion $[72,73]$

$$
\Delta_{\text {Konishi }}=2+\frac{12}{(4 \pi)^{2}} \lambda-\frac{48}{(4 \pi)^{4}} \lambda^{2}+\frac{336}{(4 \pi)^{6}} \lambda^{3}+\frac{-2496+576 \zeta(3)-1440 \zeta(5)}{(4 \pi)^{8}} \lambda^{4}+O\left(\lambda^{5}\right) .
$$

The non-perturbative methods to describe the dimension of this operator as a function of $\lambda$ using the exact integrability were developed in $[74,75]$. They have been used to develop the strong coupling expansion [76-79]

$\Delta_{\text {Konishi }}=2 \lambda^{1 / 4}-2+2 \lambda^{-1 / 4}+\left(\frac{1}{2}-3 \zeta(3)\right) \lambda^{-3 / 4}+\left(\frac{15}{2} \zeta(5)+6 \zeta(3)+\frac{1}{2}\right) \lambda^{-5 / 4}+O\left(\lambda^{-7 / 4}\right)$.

In fact, the growth of dimensions of unprotected operators as $\lambda^{1 / 4}$ was one of the first predictions of the AdS/CFT correspondence [7]. The massive closed superstring states have $m^{2}=\frac{4 n}{\alpha^{\prime}}, n=1, \ldots$. The dimension of operator dual to such a massive string in $A d S_{5} \times S^{5}$ is

$$
\Delta=2+\sqrt{4+(m L)^{2}}=2 \sqrt{n} \lambda^{1 / 4}+\ldots, \quad \lambda \gg 1 .
$$

The Konishi operator is dual to the first massive state, $n=1$, and this explains why the coefficient of the first term in (2.16) is 2. The next three terms in the strong coupling expansion (2.16) also agree with calculations $[80,81]$ using superstring theory in $A d S_{5} \times S^{5}$. Thus, studies of the scaling dimension of the Konishi operator in the large $N$ limit of the $\mathscr{N}=4$ supersymmetric $S U(N)$ gauge theory have led to highly non-trivial tests of the AdS/CFT correspondence.

\section{Vector, Matrix and Tensor Models: Snails vs. Melons}

While in the previous sections we gave a broad survey of the history and relevance of various large $N$ limits, in this section we will focus on the comparison of three "basic" large $N$ limits. Two of them, the vector and matrix large $N$ limits are widely known and have been studied for many years. The third applies only to theories with tensor degrees of freedom of rank 3 and higher. For such tensor theories with specially chosen interactions, it can be shown that the diagrammatic expansion of the path integral is dominated by the so-called "melonic" graphs. The two-loop melon 
propagator correction (better known as the sunset graph) in $\phi^{4}$ theory is shown in the right part of figure 2. The different large $N$ limits are characterized by the competition of this diagram with the one-loop snail diagram, shown in the left part of that figure.

We call these three limits basic because their existence can be shown through combinatorial analysis alone and does not hinge on specific dimensionality or additional symmetries of the theory. Therefore, instead of $d$-dimensional QFT we will first consider the $d=0$ examples, which are simply integrals. These examples also provide good practice for deriving the symmetry factors of various Feynman diagrams.

\section{1 $\phi^{4}$ models in $d=0$}

As a warm-up let us consider a one-field example, which is the $d=0 \phi^{4}$ theory. Here the partition function is simply an integral over one real variable:

$$
Z(g)=\int_{-\infty}^{\infty} \frac{d \phi}{\sqrt{2 \pi}} e^{-\frac{\phi^{2}}{2}-g \frac{\phi^{4}}{24}}
$$

This integral may be expanded in powers of $g$ using the integral

$$
I_{n}=\int_{-\infty}^{\infty} \frac{d \phi}{\sqrt{2 \pi}}\left(\phi^{2} / 2\right)^{n} e^{-\alpha \phi^{2} / 2}=(-1)^{n} \partial_{\alpha}^{n} \alpha^{-1 / 2}
$$

giving

$$
Z(g)=1-\frac{g}{8}+\frac{35 g^{2}}{384}-\frac{385 g^{3}}{3072}+\mathscr{O}\left(g^{4}\right) .
$$

In fact, in this simple example the integral may be evaluated exactly:

$$
Z(g)=\sqrt{\frac{3}{2 \pi g}} e^{\frac{3}{4 g}} K_{1 / 4}\left(\frac{3}{4 g}\right),
$$

where $K_{\alpha}(x)$ is the modified Bessel function.

The Feynman rules corresponding to (3.1) assign the factor 1 to the propagator and $-g$ to the quartic vertex. The "free energy" $\log Z$ should be given by expansion in connected vacuum amplitudes. The only diagram appearing at order $g$ is the "figure eight" graph which has symmetry factor $1 / 8$. This graph may also be thought of as a snail diagram with two legs connected.
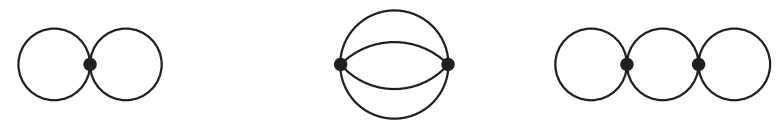

Figure 5: The three vacuum diagrams up to order $g^{2}$ : "figure eight," "melon," and "triple bubble."

At order $g^{2}$ there are two contributing connected graphs: the melon and the triple bubble graph. In the melon the two vertices are connected by 4 lines, and we find the symmetry factor $\frac{1}{2 \cdot 4 !}=\frac{1}{48}$. The bubble graph may be thought of as two snail diagrams with their legs connected, and it has symmetry factor $\frac{1}{2^{4}}=\frac{1}{16}$. Adding up these connected vacuum graphs, we therefore find

$$
\log Z(g)=-\frac{g}{8}+\frac{g^{2}}{48}+\frac{g^{2}}{16}+\mathscr{O}\left(g^{3}\right)=-\frac{g}{8}+\frac{g^{2}}{12}+\mathscr{O}\left(g^{3}\right)
$$


This agrees with the expansion of the logarithm of the exact result (3.4):

$$
\log Z(g)=-\frac{g}{8}+\frac{g^{2}}{12}-\frac{11 g^{3}}{96}+\frac{17 g^{4}}{72}-\frac{619 g^{5}}{960}+\frac{709 g^{6}}{324}-\frac{858437 g^{7}}{96768}+\mathscr{O}\left(g^{8}\right) .
$$

We note that the coefficients alternate in sign but their magnitude grows rapidly. From the properties of Bessel functions we know that at high orders it grows as $n !$. Therefore, the small $g$ expansion is only asymptotic. Nevertheless, at small $g$ inclusion of the first few orders reproduces the exact result with good precision.

Now let us extend this discussion to the case of multiple real variables $\phi^{i}$ where $i=1, \ldots, n$. The partition function may in general be written as

$$
Z=\prod_{i=1}^{n} \int_{-\infty}^{\infty} \frac{d \phi_{i}}{\sqrt{2 \pi}} \exp \left(-\frac{1}{2} \phi^{i} \phi^{i}-\frac{1}{24} C_{i j k l} \phi^{i} \phi^{j} \phi^{k} \phi^{l}\right),
$$

where $C_{i j k l}$ is a fully symmetric tensor. Various particular models may be obtained by imposing special symmetries on this tensor.

Using the connected vacuum Feynman graphs with propagator

$$
\left\langle\phi^{i} \phi^{j}\right\rangle=\delta^{i j}
$$

we obtain the following general expansion:

$$
\log Z=-\frac{C_{i i j j}}{8}+\frac{C_{i j k l} C_{i j k l}}{48}+\frac{C_{i i k l} C_{j j k l}}{16}+\mathscr{O}\left(C^{3}\right) .
$$

The first term comes from the figure eight diagram; the second from the melon; and the third from the tripple bubble.

\subsection{Vector of $O(N)$}

Let us set $n=N$ and impose the $O(N)$ symmetry on the model. Then $\phi^{i}, i=1,2, \ldots N$ transforms in the fundamental representation, and we take

$$
C_{i j k l}=\frac{g}{3}\left(\delta_{i j} \delta_{k l}+\delta_{i k} \delta_{j l}+\delta_{i l} \delta_{j k}\right)
$$

which turns the model into

$$
Z^{\mathrm{vector}}(g)=\prod_{i=1}^{N} \int_{-\infty}^{\infty} \frac{d \phi_{i}}{\sqrt{2 \pi}} e^{-\phi^{i} \phi^{i} / 2-g\left(\phi^{i} \phi^{i}\right)^{2} / 24} .
$$

From (3.9) we obtain the expansion

$$
\frac{\log Z^{\text {vector }}(g)}{N}=-\frac{N+2}{24} g+\frac{N+2}{144} g^{2}+\frac{(N+2)^{2}}{144} g^{2}+\mathscr{O}\left(g^{3}\right),
$$

where the second term comes from the melon graph, and the third from the bubble graph. To insure that $\frac{\log Z^{\text {vector }}(g)}{N}$ is finite in the large $N$ limit, we must keep $\lambda=g N$ fixed. Then the melon graph is suppressed by $1 / N$ while the bubble graphs, which originate from snail diagrams, survive. In fact, by drawing the index structure of the graphs it is not hard to see that the only surviving ones involve chains of bubbles. Thus, in vector models, the snails beat the melons. 
In the large $N$ limit the free energy has the structure

$$
\frac{\log Z^{\text {vector }}(g)}{N}=f_{0}(\lambda)+N^{-1} f_{1}(\lambda)+\ldots
$$

where $f_{0}(\lambda)=-\lambda / 24+\lambda^{2} / 144+\mathscr{O}\left(\lambda^{3}\right)$. The function $f_{0}(\lambda)$ may be determined non-perturbatively using the standard method of introducing an auxiliary variable $\sigma$, so that

$$
Z^{\text {vector }}(g)=\int_{-\infty}^{\infty} \prod_{j=1}^{N} \frac{d \phi_{j}}{\sqrt{2 \pi}} \int d \sigma \sqrt{\frac{6}{\pi g}} \exp \left(-\frac{6 N \sigma^{2}}{\lambda}-\frac{\phi^{k} \phi^{k}(1+2 i \sigma)}{2}\right) .
$$

After performing the Gaussian integral over $\phi^{j}$ we find

$$
Z^{\mathrm{vector}}(g)=\sqrt{\frac{6}{\pi g}} \int d \sigma \exp \left(-\frac{6 N \sigma^{2}}{\lambda}-\frac{N}{2} \log (1+2 i \sigma)\right) .
$$

For large $N$ the integral is dominated by the saddle point located at $\sigma=-i \tilde{\sigma}$ where

$$
\frac{12 \tilde{\sigma}}{\lambda}=\frac{1}{1+2 \tilde{\sigma}} .
$$

The solution of this quadratic equation which matches onto the perturbation theory is

$$
\tilde{\sigma}(\lambda)=\frac{\sqrt{1+\frac{2 \lambda}{3}}-1}{4}
$$

and we find

$$
f_{0}^{\text {vector }}(\lambda)=\frac{6 \tilde{\sigma}^{2}}{\lambda}-\frac{1}{2} \log (1+2 \tilde{\sigma})=-\frac{\lambda}{24}+\frac{\lambda^{2}}{144}-\frac{5 \lambda^{3}}{2592}+\frac{7 \lambda^{4}}{10368}-\frac{7 \lambda^{5}}{25920}+\mathscr{O}\left(\lambda^{6}\right),
$$

which agrees with our Feynman graph calculations. To all orders in $\lambda$,

$$
f_{0}^{\mathrm{vector}}(\lambda)=\sum_{k=1}^{\infty}(-\lambda)^{k} \frac{1}{4 k(k+1) 6^{k}}\left(\begin{array}{c}
2 k \\
k
\end{array}\right) .
$$

In this series the coefficients decrease, so it is convergent for sufficiently small $|\lambda|$. This is one of the advantages of the large $N$ limit - the functions that appear order by order in $1 / N$ have perturbation series with a finite radius of convergence.

Let us note a remarkable fact: $f_{0}(\lambda)$ makes sense even for negative $\lambda$, so long as it is greater than $\lambda_{c}=-3 / 2$ (for $\lambda<\lambda_{c}$ it is ambiguous due a branch cut). Thus, a large $N$ limit may be defined even for potentials that are not bounded from below. The expansion for $\lambda>\lambda_{c}$ is

$$
f_{0}^{\mathrm{vector}}(\lambda)=\frac{2 \log 2-1}{4}-\frac{1}{6}\left(\lambda-\lambda_{c}\right)+\frac{2}{9} \sqrt{\frac{2}{3}}\left(\lambda-\lambda_{c}\right)^{3 / 2}+\mathscr{O}\left(\left(\lambda-\lambda_{c}\right)^{2}\right) .
$$

It is common to parametrize the leading singular term as $\left(\lambda-\lambda_{c}\right)^{2-\gamma}$, and $\gamma$ is called the susceptibility exponent. We find $\gamma_{\text {vector }}=1 / 2$, which is characteristic of the branched polymers [82]. 


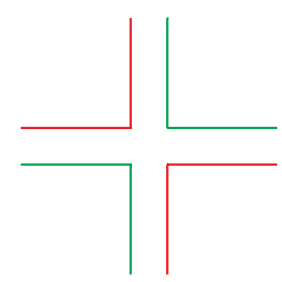

Figure 6: The resolved quartic vertex of the real matrix model (3.22).

\section{3 $O(N) \times O(N)$ symmetric real matrix model}

Now let us consider $n=N^{2}$ real degrees of freedom $\phi^{a b}, a, b=1, \ldots, N$, and impose $O(N) \times$ $O(N)$ symmetry, so that $\phi^{a b}$ are in the bi-fundamental representation. The two indices of the matrix are distinguishable, and each one is acted on by a different $O(N)$ group:

$$
\phi^{a b}=M_{1}^{a a^{\prime}} M_{2}^{b b^{\prime}} \phi^{a^{\prime} b^{\prime}}, \quad M_{1} \in O(N)_{1}, \quad M_{2} \in O(N)_{2} .
$$

We will study the matrix integral

$$
Z^{\text {matrix }}(g)=\prod_{a, b} \int_{-\infty}^{\infty} \frac{d \phi^{a b}}{\sqrt{2 \pi}} \exp \left(-\frac{1}{2} \phi^{a b} \phi^{a b}-\frac{g}{24} \phi^{a_{1} b_{1}} \phi^{a_{1} b_{2}} \phi^{a_{2} b_{1}} \phi^{a_{2} b_{2}}\right) .
$$

The propagator

$$
\left\langle\phi^{a_{1} b_{1}} \phi^{a_{2} b_{2}}\right\rangle=\delta^{a_{1} a_{2}} \delta^{b_{1} b_{2}},
$$

may be represented by a double line consisting of a red and a green strand, while the interaction vertex is shown in figure 6.

Using the matrix notation, we may write

$$
\phi^{a b} \phi^{a b}=\operatorname{tr}\left(\phi \phi^{T}\right), \quad \phi^{a_{1} b_{1}} \phi^{a_{1} b_{2}} \phi^{a_{2} b_{1}} \phi^{a_{2} b_{2}}=\operatorname{tr}\left(\phi \phi^{T} \phi \phi^{T}\right) .
$$

This demonstrates the invariance of the model under $\phi \rightarrow M_{1} \phi M_{2}^{T}$. There is one more $O(N) \times O(N)$ invariant quartic term:

$$
V_{d t}=\frac{g_{d t}}{24} \operatorname{tr}\left(\phi \phi^{T}\right) \operatorname{tr}\left(\phi \phi^{T}\right)
$$

To achieve a smooth large $N$ limit, the double-trace coupling has to be scaled as $g_{d t} \sim N^{-2}$, while the single-trace coupling as $g \sim N^{-1}$. In this scaling, models including double-trace couplings are tractable [83, 84], but we will not discuss them further.

We can write the single-trace term as

$$
\operatorname{tr}\left(\phi \phi^{T} \phi \phi^{T}\right)=\phi^{a_{1} b_{1}} \phi^{a_{2} b_{2}} \phi^{a_{3} b_{3}} \phi^{a_{4} b_{4}} \delta^{a_{1} a_{2}} \delta^{a_{3} a_{4}} \delta^{b_{1} b_{3}} \delta^{b_{2} b_{4}} .
$$

Appropriately symmetrizing this product of Kronecker symbols we obtain the version of tensor $C^{i j k l}$ appropriate for this model.

Using the connected vacuum Feynman graphs we obtain the expansion

$$
\frac{\log Z^{\text {matrix }}(g)}{N^{2}}=-\frac{1}{24}(2 N+1) g+\frac{1}{288}\left(N^{2}+2 N+3\right) g^{2}+\frac{1}{144}(2 N+1)^{2} g^{2}+\mathscr{O}\left(g^{3}\right),
$$


where the second term comes from the melon graph, and the third from the bubble graph. In order to keep $\frac{\log Z^{\text {matrix }}(g)}{N^{2}}$ finite, we keep $\lambda=g N$ fixed in the large $N$ limit. Now we see that both the melon and bubble graphs contribute at leading order. Thus, in matrix models, the competition between snails and melons results in a draw.
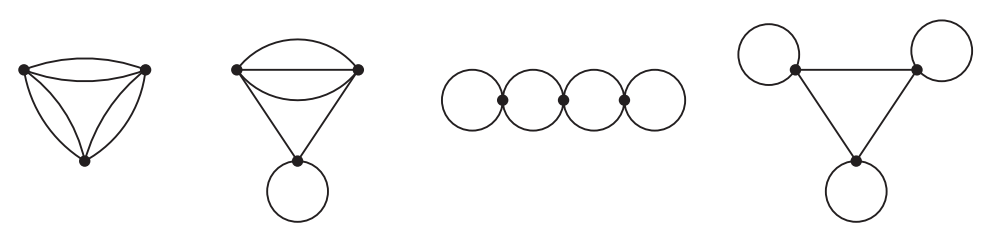

Figure 7: Third order vacuum diagrams.

Continuing to the diagrams of order $g^{3}$, we find

$$
\begin{aligned}
\left.\frac{\log Z^{\text {matrix }}(g)}{N^{2}}\right|_{g^{3} \text { order }}= & -\frac{1}{48} \cdot \frac{1}{27}\left(N^{3}+4 N^{2}+13 N+9\right) g^{3}-\frac{1}{24} \cdot \frac{1}{18}(2 N+1)\left(N^{2}+2 N+3\right) g^{3} \\
& -\frac{1}{32} \cdot \frac{1}{27}(2 N+1)^{3} g^{3}-\frac{1}{48} \cdot \frac{1}{27}(2 N+1)^{3} g^{3}
\end{aligned}
$$

where the four terms correspond to the four diagrams shown in Figure 7, respectively. All of them contribute in the large $N$ limit. In fact, 't Hooft proved [4] that all the planar graphs, i.e. the graphs of spherical topology, are dominant. To demonstrate this, it is convenient to rescale the matrix $\phi^{a b} \rightarrow \sqrt{N} \phi^{a b}$. Then

$$
Z^{\text {matrix }} \sim \prod_{a, b} \int_{-\infty}^{\infty} \frac{d \phi^{a b}}{\sqrt{2 \pi}} \exp \left(-\frac{N}{2} \phi^{a b} \phi^{a b}-\frac{N \lambda}{24} \phi^{a_{1} b_{1}} \phi^{a_{1} b_{2}} \phi^{a_{2} b_{1}} \phi^{a_{2} b_{2}}\right) .
$$

Now each propagator carries a factor $1 / N$, and each vertex factor $N \lambda$. Also, each face of the graph contains an index loop and contributes a factor of $N$. So, the net power of $N$ for a Feynman graph with $V$ vertices, $F$ faces and $E$ edges is $N^{V+F-E}=N^{\chi}$, where $\chi$ is the Euler characteristic. Since $\chi=2-2 g$, where $g$ is the genus of the graph, we see that the graphs contributing at order $N^{2}$ are the graphs of genus 0 , i.e. of spherical topology.

In the large $N$ limit the free energy (3.27) has the structure

$$
\frac{\log Z^{\text {matrix }}(g)}{N^{2}}=f_{0}^{\text {matrix }}(\lambda)+N^{-1} f_{1 / 2}^{\text {matrix }}(\lambda)+N^{-2} f_{1}^{\text {matrix }}(\lambda)+\ldots
$$

where $f_{g}(\lambda)$ is the sum over graphs of genus $g$. We see that the leading correction is due to the non-orientable surfaces of genus $1 / 2$, which is $R P_{2}$. For the Hermitian matrix model, discussed in section 3.4, such non-orientable surfaces of odd Euler characteristic do not appear. Our perturbative calculation gives $f_{0}^{\text {matrix }}(\lambda)=-\lambda / 12+\lambda^{2} / 32-\lambda^{3} / 48+\mathscr{O}\left(\lambda^{4}\right)$.

Let us obtain the exact expression for $f_{0}^{\text {matrix }}(\lambda)$, which is analogous to the one we obtained for the vector model. To do this we represent the real $N \times N$ matrix using the singular value decomposition:

$$
\phi=L \kappa R^{T},
$$


where $\kappa$ is a diagonal matrix of real non-negative singular values $\kappa_{a}$, and $R$ and $L$ are two independent $O(N)$ matrices. Integrating them out, we find

$$
Z^{\text {matrix }}(g) \sim \prod_{a} \int_{0}^{\infty} d \kappa_{a}\left|\Delta\left(\kappa^{2}\right)\right| e^{-N \sum_{b=1}^{N}\left(\frac{1}{2} \kappa_{b}^{2}+\frac{\lambda}{24} \kappa_{b}^{4}\right)},
$$

where $\Delta\left(\kappa^{2}\right)=\prod_{a<b}\left(\kappa_{a}^{2}-\kappa_{b}^{2}\right)$ is the Vandermonde determinant. A way to understand this form of the Jacobian is to note that $\kappa_{a}^{2}$ are the eigenvalues of the real symmetric matrix $\phi^{T} \phi$. Introducing the singular value density $\rho(\kappa)$,

$$
\int_{0}^{\infty} d \kappa \rho(\kappa)=1
$$

we see that in the large $N$ limit it is governed by the effective potential

$$
V_{e f f}=\int_{0}^{\infty} d \kappa \rho(\kappa)\left(\frac{1}{2} \kappa^{2}+\frac{\lambda}{24} \kappa^{4}\right)-\frac{1}{2} \int_{0}^{\infty} d \kappa d \kappa^{\prime} \rho(\kappa) \rho\left(\kappa^{\prime}\right) \log \left|\kappa^{2}-\left(\kappa^{\prime}\right)^{2}\right|
$$

Now it is convenient $[85,86]$ to introduce the symmetric function $\tilde{\rho}(\kappa)=(\rho(\kappa)+\rho(-\kappa)) / 2$, which is defined on the entire real axis, so that

$$
V_{e f f}=\int_{-\infty}^{\infty} d \kappa \tilde{\rho}(\kappa)\left(\frac{1}{2} \kappa^{2}+\frac{\lambda}{24} \kappa^{4}\right)-\int_{-\infty}^{\infty} d \kappa d \kappa^{\prime} \tilde{\rho}(\kappa) \tilde{\rho}\left(\kappa^{\prime}\right) \log \left|\kappa-\kappa^{\prime}\right|
$$

The singular integral equation which follows from this was solved in [16]:

$$
\begin{aligned}
& \tilde{\rho}(\kappa)=\frac{1}{\pi}\left(\frac{1}{2}+\frac{\lambda}{6} a^{2}+\frac{\lambda}{12} \kappa^{2}\right) \sqrt{4 a^{2}-\kappa^{2}}, \\
& a^{2}(\lambda)=\frac{\sqrt{1+2 \lambda}-1}{\lambda} .
\end{aligned}
$$

We see that $\tilde{\rho}(\kappa)$ is a symmetric function with support between $-2 a(\lambda)$ and $2 a(\lambda)$. We finally have

$$
\begin{array}{ll}
\rho(\kappa)=2 \tilde{\rho}(\kappa), & \kappa>0, \\
\rho(\kappa)=0, & \kappa<0 .
\end{array}
$$

As $\lambda \rightarrow 0, a \rightarrow 1$, and it approaches the classic Wigner semicircle law found for the Gaussian matrix models. The non-Gaussian effects deform the density to the more general function (3.36). Substituing (3.36) into $V_{\text {eff }}$ we find

$$
f_{0}^{\text {matrix }}(\lambda)=\frac{1}{24}\left(a^{2}(\lambda)-1\right)\left(9-a^{2}(\lambda)\right)-\frac{1}{2} \log a^{2}(\lambda)=-\frac{\lambda}{12}+\frac{\lambda^{2}}{32}-\frac{\lambda^{3}}{48}+\frac{7 \lambda^{4}}{384}+\mathscr{O}\left(\lambda^{5}\right) .
$$

Similarly to the free energy in the vector case, $f_{0}^{\text {matrix }}(\lambda)$ is well defined for $\lambda>\lambda_{c}$ where $\lambda_{c}=$ $-1 / 2$. Expanding $f_{0}^{\text {matrix }}(\lambda)$ near $\lambda_{c}$ we find that the leading singular term is now $\sim\left(\lambda-\lambda_{c}\right)^{5 / 2}$ corresponding to $\gamma_{\text {matrix }}=-1 / 2$. This is the well-known susceptibility exponent of the pure twodimensional quantum gravity [17-19]. In the limit $\lambda \rightarrow \lambda_{c}$ the discretized random square lattices, which are the dual lattices to the Feynman graphs for the matrix integral (3.29), become large (a section of such a lattice is shown in figure 8). Therefore, in this limit it is possible to define the continuum limit of two-dimensional quantum gravity. 


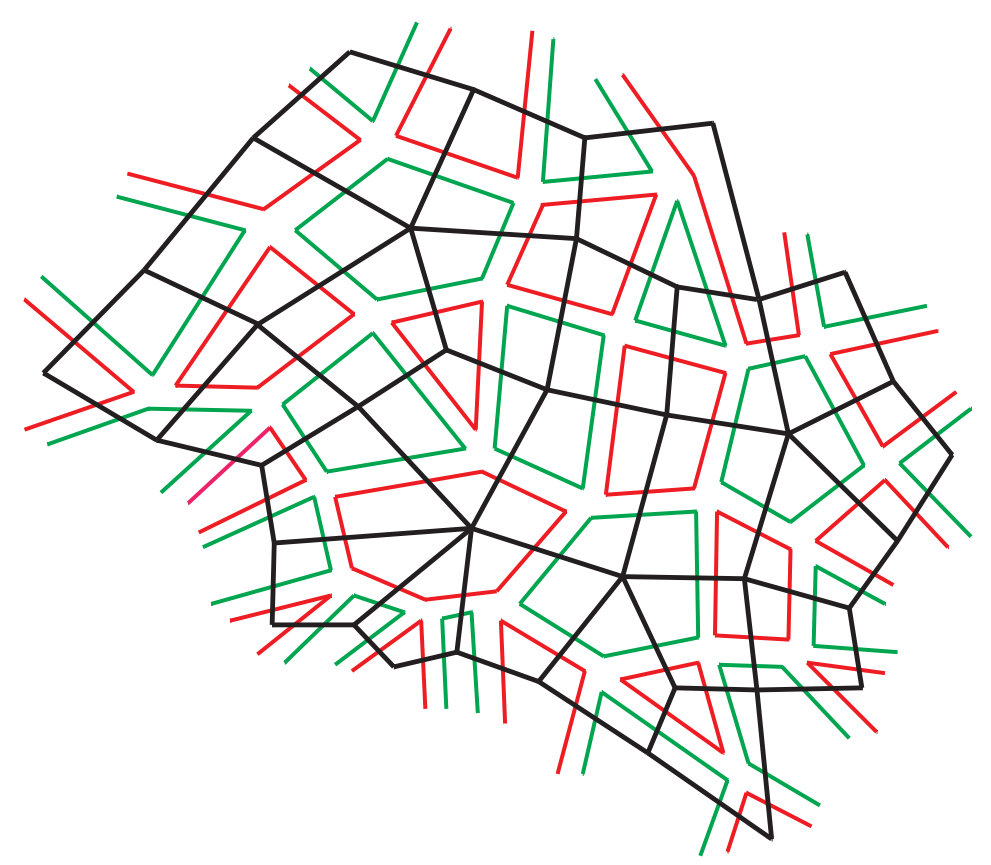

Figure 8: A secton of a resolved Feynman graph for the real matrix model, which has alternating red and green loops. Its dual lattice is made of randomly connected squares.

\section{4 $S U(N)$ symmetric Hermitian matrix model}

Now let us consider a somewhat different matrix integral. It involves a Hermitian matrix $\Phi_{j}^{i}$, $i, j=1, \ldots, N$, and we impose $S U(N)$ symmetry. $\Phi$ is in the adjoint representation, i.e. it transforms as

$$
\Phi=U \Phi^{\prime} U^{\dagger}
$$

where $U \in S U(N)$. An interesting integral to consider is

$$
Z^{\text {Hermitian }}(g)=\prod_{i, j} \int_{-\infty}^{\infty} \frac{d \operatorname{Re} \Phi_{j}^{i}}{\sqrt{2 \pi}} \frac{d \operatorname{Im} \Phi_{j}^{i}}{\sqrt{2 \pi}} \exp \operatorname{tr}\left(-\frac{1}{2} \Phi^{2}-\frac{g_{3}}{6} \Phi^{3}\right)
$$

This may be viewed as a toy model for interactions of gluons, and the large $N$ limit is taken keeping $\lambda=g_{3}^{2} N$ fixed.

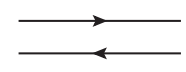

Figure 9: Hermitian matrix propagator in the double line representation.

The propagator

$$
\left\langle\Phi_{j_{1}}^{i_{1}} \Phi_{j_{2}}^{i_{2}}\right\rangle=\delta_{j_{2}}^{i_{1}} \delta_{j_{1}}^{i_{2}},
$$

may be represented using double lines with opposite directions (see Figure 9). The graphs dual to the Feynman graphs are now made of triangles, so that this integral may be interpreted in terms of 
orientable triangulated surfaces (see Figure 10). If we also impose the condition that $\Phi$ is traceless, so that it is truly in the adjoint representation of $S U(N)$, then the propagator becomes

$$
\left\langle\Phi_{j_{1}}^{i_{1}} \Phi_{j_{2}}^{i_{2}}\right\rangle=\delta_{j_{2}}^{i_{1}} \delta_{j_{1}}^{i_{2}}-\frac{1}{N} \delta_{j_{1}}^{i_{1}} \delta_{j_{2}}^{i_{2}}
$$

The tracelessness condition removes some of the tadpole graphs.

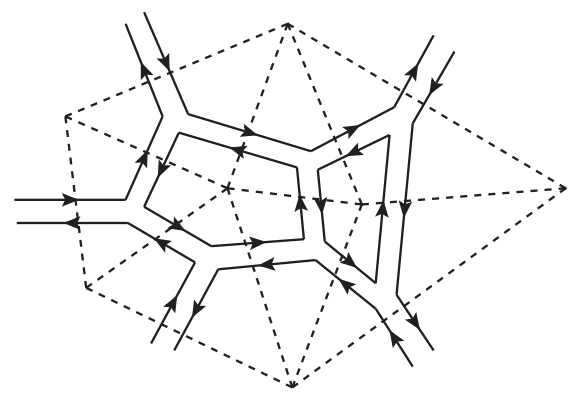

Figure 10: A section of a planar diagram in the Hermitian matrix model, which represents an orientable triangulated random surface.

We can decompose a Hermitian matrix as $\Phi=V \kappa V^{\dagger}$, where $\kappa$ is a diagonal matrix of real eigenvalues $\kappa_{a}$, which add up to zero if $\Phi$ is traceless, and $V$ is an $S U(N)$ matrix. Integrating over $V$ gives [16]

$$
Z^{\text {Hermitian }}(g) \sim \prod_{a} \int_{-\infty}^{\infty} d \kappa_{a} \Delta^{2}(\kappa) \exp \left(-\sum_{b=1}^{N}\left(\frac{1}{2} \kappa_{b}^{2}+\frac{g_{3}}{6} \kappa_{b}^{3}\right)\right) .
$$

Now, the critical behavior is $f_{0}(\lambda) \sim\left(\lambda_{c}-\lambda\right)^{5 / 2}$ [16], which is again characterized by the susceptibility exponent $\gamma_{\text {matrix }}=-1 / 2$.

\section{5 $O(N)^{3}$ symmetric real tensor model}

Now let us consider $N^{3}$ real degrees of freedom $\phi^{a b c}, a, b, c=1, \ldots, N$, and impose $O(N)^{3}$ symmetry, so that $\phi^{a b c}$ are in the tri-fundamental representation. ${ }^{2}$ The 3 indices of a tensor are distinguishable, and each one is acted on by a different $O(N)$ group:

$$
\begin{aligned}
& \phi^{a b c}=M_{1}^{a a^{\prime}} M_{2}^{b b^{\prime}} M_{3}^{c c^{\prime}} \phi^{a^{\prime} b^{\prime} c^{\prime}}, \\
& M_{1} \in O(N)_{1}, \quad M_{2} \in O(N)_{2}, \quad M_{3} \in O(N)_{3} .
\end{aligned}
$$

The most general $O(N)^{3}$ invariant quartic potential has the form

$$
\begin{aligned}
& V_{4}=\frac{g}{24} \phi^{a_{1} b_{1} c_{1}} \phi^{a_{1} b_{2} c_{2}} \phi^{a_{2} b_{1} c_{2}} \phi^{a_{2} b_{2} c_{1}}+ \\
& \quad \frac{g_{p 1}}{24} \phi^{a_{1} b_{1} c_{1}} \phi^{a_{2} b_{1} c_{1}} \phi^{a_{2} b_{2} c_{2}} \phi^{a_{1} b_{2} c_{2}}+\frac{g_{p 2}}{24} \phi^{a_{1} b_{1} c_{1}} \phi^{a_{1} b_{2} c_{1}} \phi^{a_{2} b_{1} c_{2}} \phi^{a_{2} b_{2} c_{2}}+\frac{g_{p 3}}{24} \phi^{a_{1} b_{1} c_{1}} \phi^{a_{1} b_{1} c_{2}} \phi^{a_{2} b_{2} c_{2}} \phi^{a_{2} b_{2} c_{1}} \\
& \quad+\frac{g_{d s}}{24}\left(\phi^{a_{1} b_{1} c_{1}} \phi^{a_{1} b_{1} c_{1}}\right)^{2} .
\end{aligned}
$$

${ }^{2} \mathrm{~A}$ natural generalization is to have $a=1, \ldots, N_{1}, b=1, \ldots, N_{2}, c=1, \ldots, N_{3}$, leading to a tensor model with $O\left(N_{1}\right) \times O\left(N_{2}\right) \times O\left(N_{3}\right)$ symmetry. Then $\phi^{a b c}$ may be thought of as a collection of $N_{2}$ matrices, each one $N_{1} \times N_{3}$. A limit where $N_{2}$ is taken to infinity first was studied in [87,88]. 
The first is the "tetrahedral" quartic term [32,34], which is the leftmost diagram in figure 12; the next three are the so-called pillow terms which are the remaining three diagrams in the figure. The final term is the double-sum term. We will be interested in the large $N$ limit where the tetrahedral coupling is dominant and scales as $g \sim N^{-3 / 2}$, while the remaining couplings scale to zero faster: $g_{p} \sim N^{-2}$, and $g_{d s} \sim N^{-3}[32,89]$. Therefore, we will include $g$ only and study the integral

$$
Z^{\text {tensor }}(g)=\prod_{a, b, c} \int_{-\infty}^{\infty} \frac{d \phi^{a b c}}{\sqrt{2 \pi}} \exp \left(-\frac{1}{2} \phi^{a b c} \phi^{a b c}-\frac{g}{24} \phi^{a_{1} b_{1} c_{1}} \phi^{a_{1} b_{2} c_{2}} \phi^{a_{2} b_{1} c_{2}} \phi^{a_{2} b_{2} c_{1}}\right) .
$$

Even though this quartic term is not bounded from below for $N>2$, it is possible to develop formal perturbative expansion in $g$ using the propagator

$$
\left\langle\phi^{a_{1} b_{1} c_{1}} \phi^{a_{2} b_{2} c_{2}}\right\rangle=\delta^{a_{1} a_{2}} \delta^{b_{1} b_{2}} \delta^{c_{1} c_{2}} .
$$

For the purposes of counting the powers of $N$, we can draw the resolved (or "stranded") graphs where the strands are of three different colors, corresponding to the indices transforming under the three different $O(N)$ groups. The propagator is shown in figure 11, and the tetrahedral vertex in figure 13. The proof of melon dominance following [32,34] will be reviewed in the following section, but first let us study the low orders in perturbation theory, as we did for the vector and matrix models.

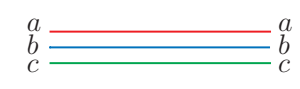

Figure 11: A resolved representation of the propagator (3.47).
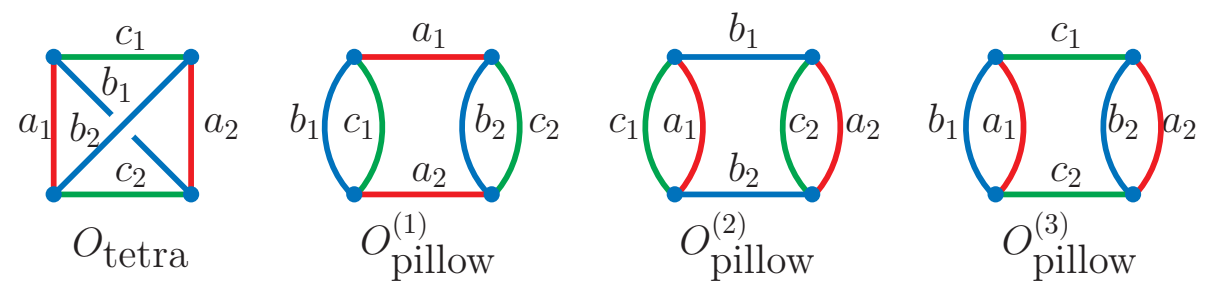

Figure 12: All the single-sum four-particle operators, the tetrahedron and the three pillows, with the index contractions shown explicitly.

Using the connected vacuum Feynman graphs we obtain the expansion

$$
\frac{\log Z^{\text {tensor }}(g)}{N^{3}}=-\frac{1}{8} N g+\frac{1}{288}\left(N^{3}+3 N+2\right) g^{2}+\frac{1}{16} N^{2} g^{2}+\mathscr{O}\left(g^{3}\right),
$$

where the first term comes from the figure eight, the second from the melon, and the third from the triple bubble graph. In order to keep $\frac{\log Z^{\text {tensor }}(g)}{N^{3}}$ finite, we keep $\lambda=g N^{3 / 2}$ fixed in the large $N$ limit. Now we see that the melon contributes while the figure eight and triple bubble graphs are suppressed. So, finally the melons are winning! Another way to see this is by comparing the melon and snail propagator corrections, whose resolved form is shown in figure 14. The melon diagram 


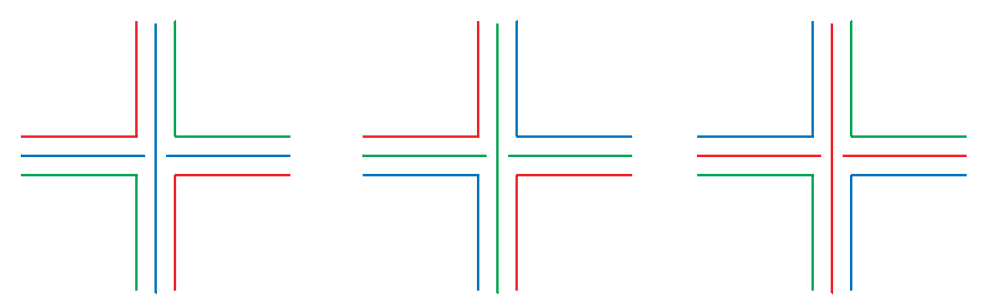

Figure 13: Three equivalent ways to represent the resolved tetrahedral vertex.
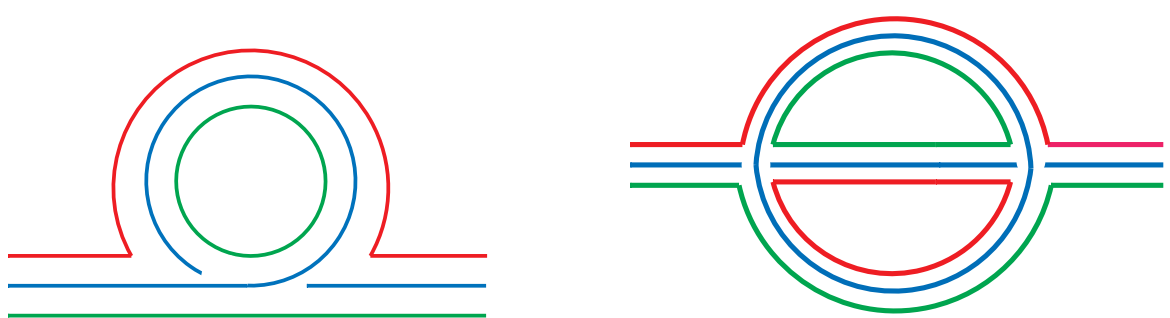

Figure 14: a) The snail propagator correction has one index loops and scales as $g N \sim \frac{\lambda}{\sqrt{N}}$, b) The melon propagator correction has three index loops and scales as $g^{2} N^{3} \sim \lambda^{2}$.

has three index loops and scales as $g^{2} N^{3} \sim \lambda^{2}$, while the snail diagram has one index loop and scales as $g N \sim \frac{\lambda}{\sqrt{N}}$.

Because of the melon dominance, in the large $N$ limit the free energy behaves as

$$
\frac{\log Z^{\text {tensor }}(g)}{N^{3}}=f_{0}^{\text {tensor }}(\lambda)+O\left(N^{-1 / 2}\right),
$$

where $f_{0}^{\text {tensor }}(\lambda)$ sums the contributions of melonic vacuum diagrams only (see figure 1 ). To solve for $f_{0}^{\text {tensor }}(\lambda)$ one can use the Schwinger-Dyson equation for the full two-point function $G(\lambda)$ implied by the diagram for self-energy [29]

$$
G^{-1}(\lambda)=1+\Sigma(\lambda), \quad \Sigma(\lambda)=-\frac{\lambda^{2}}{36} G_{\text {melons }}(\lambda)^{3} .
$$

This may be written as (see figure 15)

$$
G(\lambda)=1+\frac{\lambda^{2}}{36} G(\lambda)^{4} .
$$

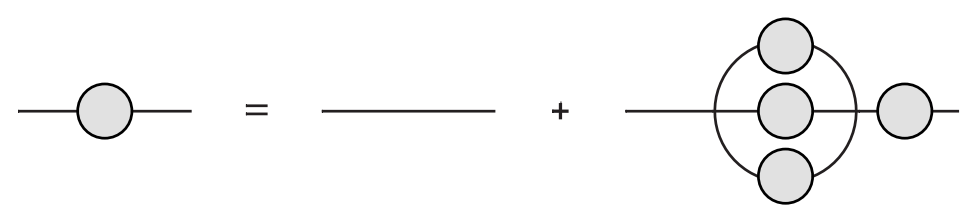

Figure 15: Schwinger-Dyson equation for the two-point function. 
Then free energy is obtained from the two-point function $G$ through the relation

$$
G(\lambda)=1+4 \lambda \partial_{\lambda} f_{0}^{\text {tensor }}(\lambda)
$$

which follows from the equation

$\frac{1}{Z^{\text {tensor }}(g)} \prod_{a, b, c} \int_{-\infty}^{\infty} \frac{d \phi^{a b c}}{\sqrt{2 \pi}} \frac{\partial}{\partial \phi^{a^{\prime} b^{\prime} c^{\prime}}}\left(\phi^{a^{\prime} b^{\prime} c^{\prime}} \exp \left(-\frac{1}{2} \phi^{a b c} \phi^{a b c}-\frac{g}{24} \phi^{a_{1} b_{1} c_{1}} \phi^{a_{1} b_{2} c_{2}} \phi^{a_{2} b_{1} c_{2}} \phi^{a_{2} b_{2} c_{1}}\right)\right)=0$.

Applying the derivative gives

$$
N^{3}-N^{3} G+\frac{4 g}{Z^{\text {tensor }}(g)} \frac{\partial}{\partial g} Z^{\text {tensor }}(g)=0,
$$

which is equivalent to (3.52).

The solution of (3.51) may be written as

$$
\begin{aligned}
& G(\lambda)=\frac{\sqrt{3}}{(2 \lambda)^{1 / 2} v^{1 / 4}}\left((1+4 v)^{1 / 4}-\left(2-(1+4 v)^{1 / 2}\right)^{1 / 2}\right) \\
& v(\lambda)=\frac{(\lambda / 3)^{2 / 3}}{2}\left[\left(1+\sqrt{1-\frac{2^{6} \lambda^{2}}{3^{5}}}\right)^{1 / 3}+\left(1-\sqrt{1-\frac{2^{6} \lambda^{2}}{3^{5}}}\right)^{1 / 3}\right] .
\end{aligned}
$$

The explicit series is $[29,90]$

$$
f_{0}^{\text {tensor }}(\lambda)=\sum_{n=1}^{\infty} a_{2 n}\left(\frac{\lambda}{6}\right)^{2 n}
$$

where

$$
a_{2}=\frac{1}{8}, \quad a_{4}=\frac{1}{4}, \quad a_{6}=\frac{11}{12}, \quad a_{8}=\frac{35}{8}, \quad \ldots, \quad a_{2 n}=\frac{1}{8 n(4 n+1)}\left(\begin{array}{c}
4 n+1 \\
n
\end{array}\right) .
$$

From the exact large $N$ solution we find that the leading singular behavior of $f_{0}^{\text {tensor }}(\lambda)$ as $\lambda$ approaches a critical value is $\left(\lambda_{c}^{2}-\lambda^{2}\right)^{3 / 2}$, where $\lambda_{c}^{2}=3^{5} / 2^{6}$, and the susceptibility exponent is $\gamma_{\text {tensor }}=1 / 2$, just as in the vector model. Therefore, the theory is again in the branched polymer phase [82].

\section{Melonic Dominance}

In this section we demonstrate the melonic dominance in theories with $O(N)^{3}$ symmetry, both in the fermionic and bosonic cases, and for any $d$. The presentation follows that in [34], and the arguments are analogous to those in [32]. We will ignore the coordinate dependence of fields and just focus on the index structure.

The propagator (3.47) has the index structure depicted in figure 11. The three colored strands (or wires) represent propagation of the three indices of the $\phi^{a b c}$ field. The tetrahedral vertex has the index structure depicted in the figure 13. There are three equivalent ways to draw the vertex; for concreteness we will use the first way. "Forgetting" the middle lines we obtain the standard matrix model vertex as in figure 6 . 

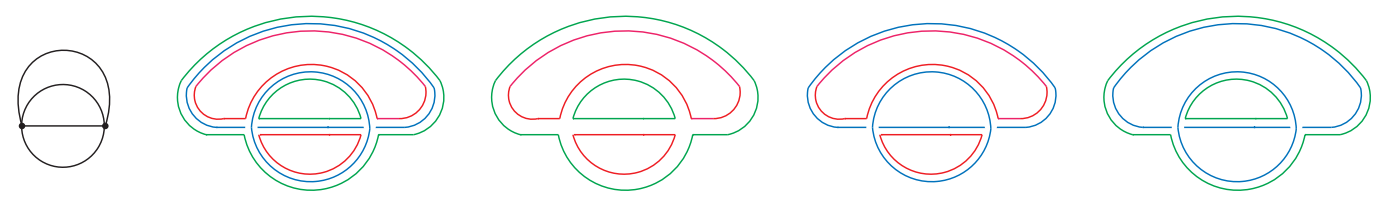

Figure 16: A melonic second-order diagram and all its double-line subgraphs.
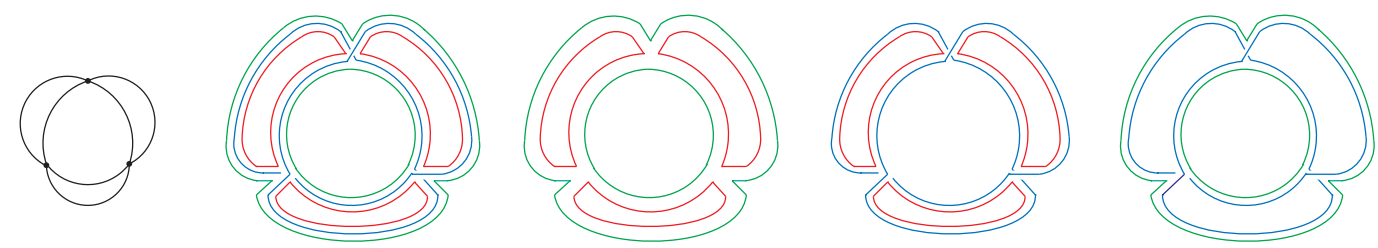

Figure 17: A non-melonic third-order diagram and all its double-line subgraphs.

Let us consider the vacuum Feynman diagrams. Examples of melonic and non-melonic diagrams with their resolved representations and double-line subgraphs are depicted in figures 16 and 17. Each resolved Feynman diagram consists of loops of three different colors and is proportional to $N^{f_{\text {total }}}$, where $f_{\text {total }}$ is the total number of index loops. Suppose we "forget" all the loops of some particular color in our diagram [33], as in figures 16 and 17. Then what remains is a double-line (or ribbon) graph of the kind one finds in matrix models. One can count the number of all index loops $f$ in this graph using the Euler characteristic $\chi$

$$
f=\chi+e-v,
$$

where $e$ is the number of edges and $v$ is the number of vertices. In our theory we obviously have $e=2 v$, therefore $f=\chi+v$. We can forget red, blue or green loops, and in each case we get a double-line graph made of the remaining two colors. If we forget, say, all red wires, then using the formula (4.1) we find $f_{b g}=\chi_{b g}+v$, where $f_{b g}=f_{b}+f_{g}$ is the number of blue and green loops and $\chi_{b g}$ is the Euler characteristic of this blue-green graph. Analogously we get $f_{r g}=\chi_{r g}+v$ and $f_{b r}=\chi_{b r}+v$. Adding up these formulas we find

$$
f_{b g}+f_{r g}+f_{b r}=2\left(f_{b}+f_{g}+f_{r}\right)=\chi_{b g}+\chi_{b r}+\chi_{r g}+3 v .
$$

Thus, the total number of loops is

$$
f_{\text {total }}=f_{b}+f_{g}+f_{r}=\frac{3 v}{2}+3-g_{b g}-g_{b r}-g_{r g},
$$

where $g=1-\chi / 2$ is the genus of a graph. Because $g \geqslant 0$ we obtain

$$
f_{\text {total }} \leqslant 3+\frac{3 v}{2} \text {. }
$$

This provides a simple proof that the maximal scaling of a vacuum graph with $v$ vertices is $\sim N^{3} \lambda^{v}$. Now the goal is to show that the equality $f_{\text {total }}=3+3 v / 2$ is satisfied only for the melonic diagrams. We will call the graphs which satisfy $f_{\text {total }}=3+3 v / 2$ the maximal graphs. Thus we should argue that maximal graphs are necessarily melonic. We note that, due to (4.3), each double-line subgraph of a maximal graph has genus zero. 
Now let us classify all loops in our graph according to how many vertices they pass through (a loop can pass the same vertex twice). Let us denote by $\mathscr{F}_{s} \geqslant 0$ the number of loops, which pass through $s$ vertices. For a maximal graph

$$
f_{\text {total }}=\mathscr{F}_{2}+\mathscr{F}_{3}+\mathscr{F}_{4}+\mathscr{F}_{5}+\ldots=3+\frac{3 v}{2},
$$

where we set $\mathscr{F}_{1}=0$. Indeed, a snail insertion into a propagator, which is the only way of obtaining an index loop of length 1 , is suppressed by a factor of $\sqrt{N}$ (see figure 14). Now, since each vertex must be passed 6 times, we also get

$$
2 \mathscr{F}_{2}+3 \mathscr{F}_{3}+4 \mathscr{F}_{4}+5 \mathscr{F}_{5}+\cdots=6 v
$$

Combining this with (4.5), we find

$$
2 \mathscr{F}_{2}+\mathscr{F}_{3}=12+\mathscr{F}_{5}+2 \mathscr{F}_{6}+\ldots
$$

Now our goal is to show that $\mathscr{F}_{2}>0$ using this formula (in fact, $\mathscr{F}_{2} \geqslant 6$, but all we will need is that it is non-vanishing).

Let us first argue that a maximal graph must have $\mathscr{F}_{3}=0$. To have $\mathscr{F}_{3}>0$ we need a closed index loop passing through 3 vertices. Without a loss of generality we can assume that this loop is formed by the middle lines in each vertex (blue lines). The only possibility with a closed loop of an internal (blue) index, which passes through three vertices, is shown in fig. 18 a). After "forgetting" the color of this loop we get the ribbon graph in fig. $18 \mathrm{~b}$ ), which is non-planar due a twisted propagator. So, a graph with $\mathscr{F}_{3}>0$ cannot be maximal. Thus, setting $\mathscr{F}_{3}=0$ in (4.7), we deduce that a maximal graph should have $\mathscr{F}_{2}>0$.
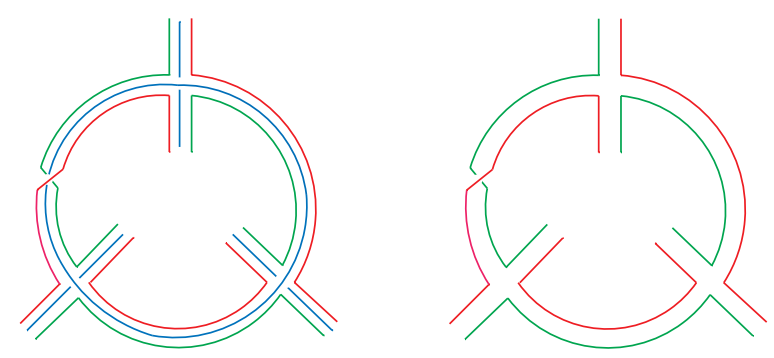

Figure 18: a) Local part of a graph with a middle index loop passing through 3 vertices. b) The same figure where the middle index has been "forgotten."

Finally, we need to show that the graphs with $\mathscr{F}_{2}>0$ are melonic. To do this we will follow Proposition 3 in [29]. Without a loss of generality we assume that the loop passing through 2 vertices is formed by the middle lines in each vertex (blue lines). The only such possibility is shown in fig. 19 a). After "forgetting" the color of this loop we get the ribbon graph in fig. 19 b). Now we uncolor the lines in our ribbon graph, and cut and sew two edges as in figure 20. We cut two edges but did not change the number of loops; therefore, the Euler characteristic of the new graph is $\chi=4$. This is possible only if we separated our original graph into two genus zero parts. 

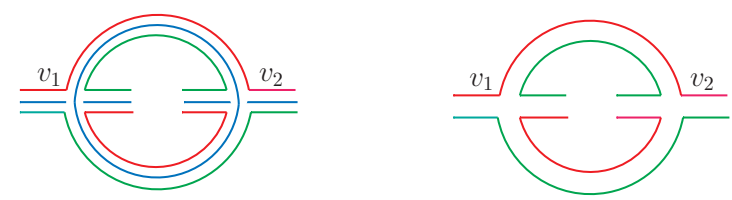

Figure 19: a) Local part of a graph with a middle index loop passing through two vertices $v_{1}$ and $v_{2}$. b) The same figure where the middle index has been "forgotten."

Therefore, our graph is two-particle reducible for the internal and external couples of lines. Thus, the whole unresolved graph looks like figure 21. Then, if graphs $G^{\prime}$ and $G^{\prime \prime}$ are empty we get a second-order melon graph as in figure 16. If they are not empty one can argue (see [29]) that they are also maximal graphs. So, we can recursively apply the same above argument to them, implying that the complete diagram is melonic.
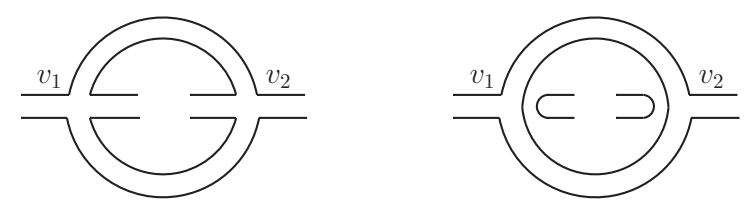

Figure 20: Cutting and sewing lines.

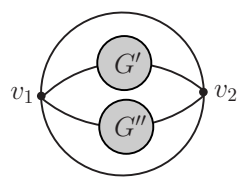

Figure 21: General structure of the maximal graph.

While the proof above applies to $O(N)^{3}$ theories, we note that the melonic dominance does not require the theory to have multiple $O(N)$ symmetry groups. Indeed, it was conjectured in [90] that the theory of a traceless symmetric or an antisymmetric 3-index bosonic tensor $\phi^{a b c}$ of $O(N)$ with the tetrahedral interaction is dominated by the melonic graphs. This conjecture was substantiated with explicit calculations up to a rather high order. The conjecture was proved in [91], but the combinatorial proof is considerably more complicated than the one presented above for the $O(N)^{3}$ theory: indeed, since in the $O(N)$ case the strands have the same color, the trick of "forgetting" all strands of a given color cannot be applied. The proof has been extended to $O(N)$ theories with rank-3 tensors of mixed symmetry [92].

\section{The Minimal $O(N)^{3}$ Tensor Quantum Mechanics}

The idea that fermionic tensor models reproduce the SYK-like large $N$ limit, but without disorder, was advanced in [33]. The quantum mechanical model constructed there, often called the Gurau-Witten model, contains four species of rank-3 tensors and has $O(N)^{6}$ symmetry. Using the $O(N)^{3}$ symmetric interaction (3.46), but replacing the real bosons with Majorana fermions $\psi^{a b c}(t)$, 
it is possible to simplify the construction of [33]. This leads to the minimal SYK-like fermionic quantum mechanical model [34] with the action

$$
S=\int d t\left(\frac{i}{2} \psi^{a b c} \partial_{t} \psi^{a b c}-\frac{1}{4} g \psi^{a_{1} b_{1} c_{1}} \psi^{a_{1} b_{2} c_{2}} \psi^{a_{2} b_{1} c_{2}} \psi^{a_{2} b_{2} c_{1}}\right),
$$

up to an additive constant. Let us emphasize that $\psi^{a b c}$ has distinguishable indices, each of which runs from 1 to $N$. At the classical level, i.e. ignoring the right-hand side of (1.2), the Fermi statistics implies

$$
\psi^{a_{1} b_{1} c_{1}} \psi^{a_{1} b_{2} c_{2}} \psi^{a_{2} b_{1} c_{2}} \psi^{a_{2} b_{2} c_{1}}=-\psi^{a_{1} b_{2} c_{2}} \psi^{a_{1} b_{1} c_{1}} \psi^{a_{2} b_{1} c_{2}} \psi^{a_{2} b_{2} c_{1}}
$$

After relabeling $b_{1} \leftrightarrow c_{2}$ and $b_{2} \leftrightarrow c_{1}$ we get the relation

$$
\psi^{a_{1} b_{1} c_{1}} \psi^{a_{1} b_{2} c_{2}} \psi^{a_{2} b_{1} c_{2}} \psi^{a_{2} b_{2} c_{1}}=-\psi^{a_{1} c_{1} b_{1}} \psi^{a_{1} c_{2} b_{2}} \psi^{a_{2} c_{2} b_{1}} \psi^{a_{2} c_{1} b_{2}}
$$

This demonstrates the vanishing of the tetrahedral interaction term in the $O(N)$ symmetric theory with fermions in any irreducible 3-index representation: fully symmetric, fully anti-symmetric or mixed symmetry.

Thus, the theory (5.1) with $O(N)^{3}$ symmetry appears to be the simplest possible tensor counterpart of the SYK model. ${ }^{3}$ The $S O(N)^{3}$ symmetry may be gauged by the replacement

$$
\partial_{t} \psi^{a b c} \rightarrow\left(D_{t} \psi\right)^{a b c}=\partial_{t} \psi^{a b c}+A_{1}^{a a^{\prime}} \psi^{a^{\prime} b c}+A_{2}^{b b^{\prime}} \psi^{a b^{\prime} c}+A_{3}^{c c^{\prime}} \psi^{a b c^{\prime}}
$$

where $A_{i}$ is the gauge field corresponding to the $i$-th $S O(N)$ group. In $d=1$ the gauge fields are non-dynamical, and their only effect is to restrict the states to be annihilated by the symmetry charges

$$
Q_{1}^{a a^{\prime}}=\frac{i}{2}\left[\psi^{a b c}, \psi^{a^{\prime} b c}\right], \quad Q_{2}^{b b^{\prime}}=\frac{i}{2}\left[\psi^{a b c}, \psi^{a b^{\prime} c}\right], \quad Q_{3}^{c c^{\prime}}=\frac{i}{2}\left[\psi^{a b c}, \psi^{a b c^{\prime}}\right] .
$$

The spectrum of the Hamiltonian (1.3) has an interesting property: for each eigenstate of energy $E$ there is a corresponding eigenstate of energy $-E$. To explain the origin of this symmetry, it is useful to introduce unitary operators $P_{i j}$ associated with permutations of the $O(N)_{i}$ and $O(N)_{j}$ groups [95]:

$$
\begin{aligned}
& P_{23}=P_{23}^{\dagger}=i^{n(n-1) / 2} \prod_{a} \prod_{b>c}\left(\psi^{a b c}-\psi^{a c b}\right), \\
& P_{12}=P_{12}^{\dagger}=i^{n(n-1) / 2} \prod_{c} \prod_{a>b}\left(\psi^{a b c}-\psi^{b a c}\right),
\end{aligned}
$$

where $n=N^{2}(N-1) / 2$ is the number of fields in the product. They satisfy

$$
P_{23} \psi^{a b c} P_{23}^{\dagger}=(-1)^{N^{2}(N-1) / 2} \psi^{a c b}, \quad P_{12} \psi^{a b c} P_{12}^{\dagger}=(-1)^{N^{2}(N-1) / 2} \psi^{b a c} .
$$

\footnotetext{
${ }^{3}$ This tensor-SYK correspondence can be generalized to the versions of SYK model where the Hamiltonian couples $q>4$ fermions. The corresponding tensor model involves a Majorana tensor with $q-1$ distinguishable indices [34,93]. The generalized tetrahedral interaction preserving $O(N)^{q-1}$ symmetry is unique for $q=6$ [34], but there is a growing set of possibilities for $q \geq 8$ [94]. The large $N$ limit is taken keeping $g^{2} N^{(q-1)(q-2) / 2}$ fixed.
} 
These permutations flip the sign of $H[54,95,96]$ :

$$
P_{23} H P_{23}^{\dagger}=-H, \quad P_{12} H P_{12}^{\dagger}=-H .
$$

Thus, if $|\Psi\rangle$ is an eigenstate of $H$ with eigenvalue $E$, then $P_{12}|\Psi\rangle$ is an eigenstate with eigenvalue $-E$.

We can further define the operator $P$ which implements a cyclic permutation of the three $O(N)$ groups:

$$
P=P_{12} P_{23}, \quad P \psi^{a b c} P^{\dagger}=\psi^{c a b} .
$$

It has the properties

$$
P H P^{\dagger}=H, \quad P^{3}=I,
$$

thus realizing the $Z_{3}$ symmetry of the Hamiltonian. For a more complete discussion of the discrete symmetries of the $O(N)^{3}$ tensor model, see [95].

A remarkable property of the model (1.3) is that, as $N$ grows, the spectrum of low-lying states becomes dense, and the theory becomes nearly conformal (we will demonstrate this in section 5.2 using the Schwinger-Dyson equations). Thus, in the large $N$ limit it is possible to define conformal operators and calculate their scaling dimensions. Particularly easy to study is the set of operators

$$
O_{2}^{n}=\psi^{a b c}\left(\partial_{t}^{n} \psi\right)^{a b c}
$$

up to a total derivative. These operators are conformal primaries when $n$ is odd. As discussed in section 5.3, using the equation of motion repeatedly, we can express them as gauge-invariant multi-particle operators without derivatives. Operators (5.11) are analogous to the "single Regge trajectory" [97-99] found in the SYK model [39-43]. In section 5.2 we will show that, in the large $N$ limit of the $O(N)^{3}$ tensor model, the scaling dimensions of these operators are the same as in the SYK model. But first let us make some comments on the relation between the tensor and SYK models.

\subsection{Comparison of the tensor and SYK Hamiltonians}

Let us compare the Hamiltonian of the $O(N)^{3}$ tensor model with that of the SYK model with $N_{\mathrm{SYK}}=N^{3}$. If we think of $I=(a b c)$ as a composite index which takes $N^{3}$ values, the Hamiltonian (1.3) may be written in a way similar to that in the Sachdev-Ye-Kitaev model: omitting the overall factor $\frac{g}{4}$,

$$
H=\frac{1}{4 !} J_{I_{1} I_{2} I_{3} I_{4}} \psi^{I_{1}} \psi^{I_{2}} \psi^{I_{3}} \psi^{I_{4}}
$$

where

$$
J_{I_{1} I_{2} I_{3} I_{4}}=\delta_{a_{1} a_{2}} \delta_{a_{3} a_{4}} \delta_{b_{1} b_{3}} \delta_{b_{2} b_{4}} \delta_{c_{1} c_{4}} \delta_{c_{2} c_{3}}-\delta_{a_{1} a_{2}} \delta_{a_{3} a_{4}} \delta_{b_{2} b_{3}} \delta_{b_{1} b_{4}} \delta_{c_{2} c_{4}} \delta_{c_{1} c_{3}}+22 \text { terms }
$$


This definition of $J$ takes values $0, \pm 1$, and it is fully antisymmetric under permutations of the indices $I_{k}=\left(a_{k} b_{k} c_{k}\right)$. Let us stress that this form of $J$ breaks the $O\left(N^{3}\right)$ symmetry of the free theory down to $O(N)^{3}$. The tensor $J$ is traceless, $J_{I_{1} I_{1} I_{2} I_{3}}=0$, and it satisfies

$$
\frac{1}{4 !} \sum_{\left\{I_{k}\right\}} J_{I_{1} I_{2} I_{3} I_{4}}^{2}=\frac{1}{4} N^{3}(N-1)^{2}(N+2) .
$$

This is the number of distinct non-vanishing terms in the Hamiltonian. ${ }^{4}$ In the SYK model with $N_{\mathrm{SYK}}=N^{3}$ fermions and random quartic couplings $J_{i_{1} i_{2} i_{3} i_{4}}$, the Hamiltonian (1.1) generally involves

$$
\frac{1}{4 !} N_{\mathrm{SYK}}\left(N_{\mathrm{SYK}}-1\right)\left(N_{\mathrm{SYK}}-2\right)\left(N_{\mathrm{SYK}}-3\right)=\frac{1}{24} N^{3}\left(N^{3}-1\right)\left(N^{3}-2\right)\left(N^{3}-3\right),
$$

distinct terms. Thus, in the $O(N)^{3}$ model almost all possible quartic couplings vanish; only a fraction of order $N^{-6}$ is non-vanishing! For example, for $N=4$, which is the biggest numerical diagonalization so far [95], the Hamiltonian contains only 864 terms out of the 635376 possible terms, which would be present in the SYK model with $N_{\mathrm{SYK}}=64$ fermions. Thus, the detailed structure of quartic couplings in the $O(N)^{3}$ tensor model is very sparse and highly non-generic from the SYK point of view. Nevertheless, the two models have similar large $N$ limits, at least for some quantities. The sparseness of the tensor model Hamiltonian facilitates applications of the Lanczos method for calculating the spectrum [95].

In the SYK model with a large number of Majorana fermion species $N_{\mathrm{SYK}}$, the energy gaps between low-lying eigenstates are of order $e^{-\alpha N_{\mathrm{SYK}}}$, where $\alpha$ is a positive constant of order 1 . This was shown numerically in $[98,100-102]$. The exponential smallness of the gaps leads to large low-temperature entropy $S_{0}=c_{0} N_{\mathrm{SYK}}$, even though the ground state is non-degenerate. The normalization constant in the $q=4$ SYK model is [98]

$$
c_{0}=\frac{1}{2} \log 2-\int_{0}^{1 / 4} \pi\left(\frac{1}{2}-x\right) \tan (\pi x) d x \approx 0.23 .
$$

In the $O(N)^{3}$ tensor model, calculation of the low-temperature entropy proceeds by summing the same melonic diagrams as in the SYK model [103]. Therefore, using $N_{\mathrm{SYK}}=N^{3}$, we find $S_{0}=c_{0} N^{3}$. By analogy with the SYK model, this suggests that the gaps above the ground states are of order $e^{-\tilde{\alpha} N^{3}}$ for large $N$, where $\tilde{\alpha}$ is a positive constant of order 1 . These tiny gaps should appear in the $S O(N)^{3}$ invariant part of the spectrum, which includes the ground state. There is a lower bound on the ground state energy [96]:

$$
E_{0}>-\frac{g}{16} N^{3}(N+2) \sqrt{N-1} .
$$

Nicely, this grows as $\lambda N^{3}$ in the large $N$ limit, just like in the SYK model the ground state energy grows as $N_{\mathrm{SYK}}$ [98]. The splittings between the $S O(N)^{3}$ non-singlet and singlet states scale to zero as $\lambda / N[96,104]$. So far, these properties of the spectrum have not been possible to demonstrate via direct numerical diagonalization of the Hamiltonian because the total size of the Hilbert space grows as $2^{N^{3} / 2}$.

\footnotetext{
${ }^{4}$ If we restore the factor $g^{2}$, then (5.14) becomes the combinatorial factor for the simplest melonic vacuum diagram, which is the leftmost in figure 1 . For large $N$ it scales as $\lambda^{2} N^{3}$, in agreement with section 4 .
} 
Explicit tensor model diagonalizations have been carried out in [95, 96, 105-107]. In the biggest calculation to date [95], the complete spectrum of $S O(4)^{3}$ invariant states was found in the $O(4)^{3}$ tensor model. Since there are only 36 such states, they do not exhibit the small gaps needed for the study of the nearly conformal behavior. However, in the $O(6)^{3}$ tensor model there are over 595 million $S O(6)^{3}$ invariant states [96], while their energies are bounded as

$$
-108 \sqrt{5} g<E<108 \sqrt{5} g .
$$

Therefore, their spectrum is likely to be very dense, but more work is needed to study their distribution.

\subsection{Schwinger-Dyson equations}

Let us study some of the diagrammatics of the $O(N)^{3}$ symmetric quantum mechanics (5.1). We will study the ungauged model; the effect of the gauging may be imposed later by restricting to the gauge invariant operators. The bare propagator is

$$
\left\langle T\left(\psi^{a b c}(t) \psi^{a^{\prime} b^{\prime} c^{\prime}}(0)\right)\right\rangle_{0}=\delta^{a a^{\prime}} \delta^{b b^{\prime}} \delta^{c c^{\prime}} G_{0}(t)=\delta^{a a^{\prime}} \delta^{b b^{\prime}} \delta^{c c^{\prime}} \frac{1}{2} \operatorname{sgn}(t) .
$$

The full propagator in the large $N$ limit receives corrections from the melonic diagrams represented in figure 22. Resummation of all melonic diagrams leads to the Schwinger-Dyson equation for the

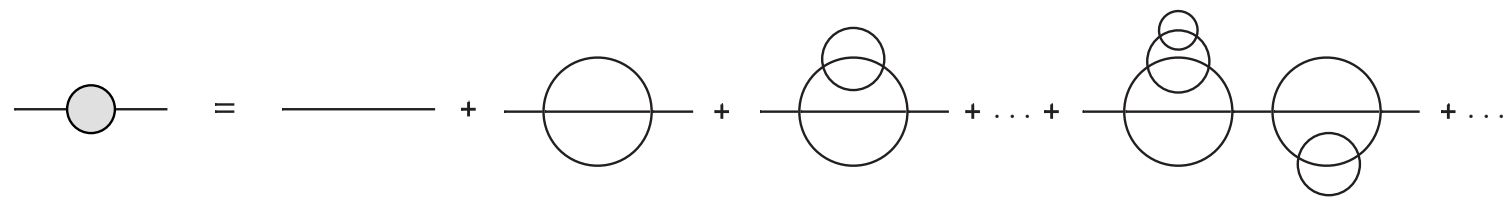

Figure 22: Diagrams contributing to the two point function in the leading large $N$ order. The line with the gray circle represents the full two point function. Each simple line is the bare propagator.

two-point function

$$
G\left(t_{1}-t_{2}\right)=G_{0}\left(t_{1}-t_{2}\right)+g^{2} N^{3} \int d t d t^{\prime} G_{0}\left(t_{1}-t\right) G\left(t-t^{\prime}\right)^{3} G\left(t^{\prime}-t_{2}\right),
$$

represented graphically in figure 15. This equation has the same structure as that derived in [97-99] for the large $N$ SYK model. The solution to (5.20) in the IR limit is

$$
G\left(t_{1}-t_{2}\right)=-\left(\frac{1}{4 \pi g^{2} N^{3}}\right)^{1 / 4} \frac{\operatorname{sgn}\left(t_{1}-t_{2}\right)}{\left|t_{1}-t_{2}\right|^{1 / 2}} .
$$

To uncover the spectrum of the bilinear operators in the model, we need to study the 4-point function $\left\langle\psi^{a_{1} b_{1} c_{1}}\left(t_{1}\right) \psi^{a_{1} b_{1} c_{1}}\left(t_{2}\right) \psi^{a_{2} b_{2} c_{2}}\left(t_{3}\right) \psi^{a_{2} b_{2} c_{2}}\left(t_{4}\right)\right\rangle$. Its structure is again the same as in the large $N$ SYK model [97,98]:

$$
\left\langle\psi^{a_{1} b_{1} c_{1}}\left(t_{1}\right) \psi^{a_{1} b_{1} c_{1}}\left(t_{2}\right) \psi^{a_{2} b_{2} c_{2}}\left(t_{3}\right) \psi^{a_{2} b_{2} c_{2}}\left(t_{4}\right)\right\rangle=N^{6} G\left(t_{12}\right) G\left(t_{34}\right)+\Gamma\left(t_{1}, \ldots, t_{4}\right),
$$

where $\Gamma\left(t_{1}, \ldots, t_{4}\right)$ is given by a series of ladder diagrams depicted in fig 23 . 


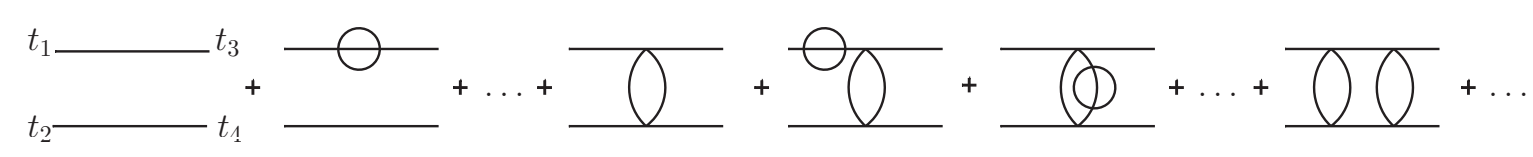

Figure 23: Ladder diagrams contributing to $\Gamma\left(t_{1}, \ldots, t_{4}\right)$

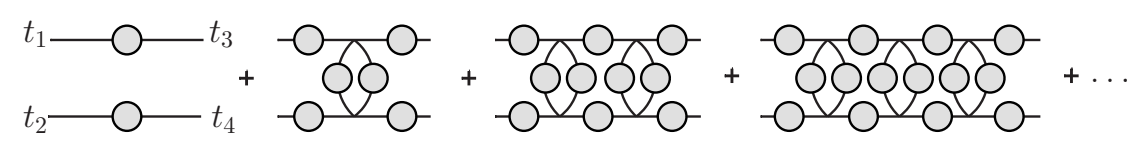

Figure 24: Ladder diagrams contributing to $\Gamma\left(t_{1}, \ldots, t_{4}\right)$

Resumming the diagrams in fig. 23 one finds a contribution to $\Gamma\left(t_{1}, \ldots, t_{4}\right)$ as a series of diagrams in terms of the full propagators, see fig. 24. If we denote by $\Gamma_{n}$ the ladder with $n$ rungs, so $\Gamma=\sum_{n} \Gamma_{n}$, we have

$$
\Gamma_{0}\left(t_{1}, \ldots, t_{4}\right)=N^{3}\left(-G\left(t_{13}\right) G\left(t_{24}\right)+G\left(t_{14}\right) G\left(t_{23}\right)\right) .
$$

For the next coefficient one gets

$$
\Gamma_{1}\left(t_{1}, \ldots, t_{4}\right)=3 g^{2} N^{6} \int d t d t^{\prime}\left(G\left(t_{1}-t\right) G\left(t_{2}-t^{\prime}\right) G\left(t-t^{\prime}\right)^{2} G\left(t-t_{3}\right) G\left(t-t_{4}\right)-\left(t_{3} \leftrightarrow t_{4}\right)\right),
$$

and one can check further that

$$
\Gamma_{2}\left(t_{1}, \ldots, t_{4}\right)=-3 g^{2} N^{3} \int d t d t^{\prime}\left(G\left(t_{1}-t\right) G\left(t_{2}-t^{\prime}\right) G\left(t-t^{\prime}\right)^{2} \Gamma_{1}\left(t, t^{\prime}, t_{3}, t_{4}\right)-\left(t_{3} \leftrightarrow t_{4}\right)\right) .
$$

So, in general, one gets exactly the same recursion relation as in the SYK model

$$
\Gamma_{n+1}\left(t_{1}, \ldots, t_{4}\right)=\int d t d t^{\prime} K\left(t_{1}, t_{2} ; t, t^{\prime}\right) \Gamma_{n}\left(t, t^{\prime}, t_{3}, t_{4}\right)
$$

where the kernel is

$$
K\left(t_{1}, t_{2} ; t_{3}, t_{4}\right)=-3 g^{2} N^{3} G\left(t_{13}\right) G\left(t_{24}\right) G\left(t_{34}\right)^{2} .
$$

In order to find the spectrum of the two-particle operators $O_{2}^{n}$, following $[98,99]$ one has to solve the integral eigenvalue equation

$$
v_{n}\left(t_{0}, t_{1}, t_{2}\right)=g_{\text {antisym }}(h) \int d t_{3} d t_{4} K\left(t_{1}, t_{2} ; t_{3}, t_{4}\right) v_{n}\left(t_{0}, t_{3}, t_{4}\right),
$$

where

$$
v_{n}\left(t_{0}, t_{1}, t_{2}\right)=\left\langle O_{2}^{n}\left(t_{0}\right) \psi^{a b c}\left(t_{1}\right) \psi^{a b c}\left(t_{2}\right)\right\rangle=\frac{c_{n} \operatorname{sgn}\left(t_{1}-t_{2}\right)}{\left|t_{0}-t_{1}\right|^{h}\left|t_{0}-t_{2}\right|^{h}\left|t_{1}-t_{2}\right|^{1 / 2-h}},
$$

is the conformal three-point function. To find $g(h)$ we compute the integral in (5.28) using general $d$ dimensional conformal integrals [108] specified to $d=1$ :

$$
\int_{-\infty}^{+\infty} d u \frac{\operatorname{sgn}\left(u-t_{1}\right) \operatorname{sgn}\left(u-t_{2}\right)}{\left|u-t_{1}\right|^{\alpha_{1}}\left|u-t_{2}\right|^{\alpha_{2}}\left|u-t_{3}\right|^{\alpha_{3}}}=l_{\alpha_{1}, \alpha_{2}} \frac{\operatorname{sgn}\left(t_{13}\right) \operatorname{sgn}\left(t_{23}\right)}{\left|t_{12}\right|^{1-\alpha_{3}}\left|t_{13}\right|^{1-\alpha_{2}}\left|t_{23}\right|^{1-\alpha_{1}}}
$$


where $\alpha_{1}+\alpha_{2}+\alpha_{3}=2$ and

$$
l_{\alpha_{1}, \alpha_{2}}=\sqrt{\pi} \frac{\Gamma\left(\frac{1-\alpha_{1}}{2}+\frac{1}{2}\right) \Gamma\left(\frac{1-\alpha_{2}}{2}+\frac{1}{2}\right) \Gamma\left(\frac{1-\alpha_{3}}{2}\right)}{\Gamma\left(\frac{\alpha_{1}}{2}+\frac{1}{2}\right) \Gamma\left(\frac{\alpha_{2}}{2}+\frac{1}{2}\right) \Gamma\left(\frac{\alpha_{3}}{2}\right)} .
$$

Taking integrals over $t_{3}$ and $t_{4}$ in (5.28) using (5.30) we find $[98,99]$

$$
g_{\text {antisym }}(h)=-\frac{3}{4 \pi} l_{\frac{3}{2}-h, \frac{1}{2}} l_{h+\frac{1}{2}, \frac{1}{2}}=-\frac{3}{2} \frac{\tan \left(\frac{\pi}{2}\left(h-\frac{1}{2}\right)\right)}{h-1 / 2} .
$$

The scaling dimensions are given by the solutions of $g_{\text {antisym }}(h)=1$. The first solution is exact, $h=2$; this is the important mode dual to gravity and responsible for the quantum chaos in the model [97, 98, 109-113]. The further solutions are $h \approx 3.77,5.68,7.63,9.60$ corresponding to operators $O_{2}^{n}$ with $n=3,5,7,9$. In the limit of large $n, h_{n} \rightarrow n+\frac{1}{2}$. This is the expected limit $n+2 \Delta$, where $\Delta=\frac{1}{4}$ is the scaling dimension of the individual fermion.

\subsection{Multi-particle operators}

The model of [34] contains a rapidly growing number of $S O(N)^{3}$ invariant (2k)-particle operators. Since a time derivative may be removed using the equations of motion, we may write the operators in a form where no derivatives are present. The bilinear singlet operator, $\psi^{a b c} \psi^{a b c}$, vanishes classically by the Fermi statistics, while at the quantum level taking into account (1.2), it is a C-number. The first non-trivial operators appear at the quartic level and are shown in figure 12. All of them are "single-sum" operators, i.e. those that correspond to connected diagrams; they cannot be written as products of invariant operators. On the left is the "tetrahedron operator" $O_{\text {tetra }}$, which appears in the Hamiltonian (1.3). The three additional operators in figure 12, which we denote as $O_{\text {pillow }}^{(1)}, O_{\text {pillow }}^{(2)}$ and $O_{\text {pillow }}^{(3)}$, are the "pillow" operators in the terminology of [30,32]; they contain double lines between a pair of vertices. Up to an additive constant,

$$
O_{\text {pillow }}^{(1)}=\sum_{a_{1}<a_{2}} Q_{1}^{a_{1} a_{2}} Q_{1}^{a_{1} a_{2}}, \quad O_{\text {pillow }}^{(2)}=\sum_{b_{1}<b_{2}} Q_{2}^{b_{1} b_{2}} Q_{2}^{b_{1} b_{2}}, \quad O_{\text {pillow }}^{(3)}=\sum_{c_{1}<c_{2}} Q_{3}^{c_{1} c_{2}} Q_{3}^{c_{1} c_{2}},
$$

i.e. they are the quadratic Casimir operators of the three $S O(N)$ groups. Since the $S O(N)^{3}$ charges (5.5) commute with the Hamiltonian (1.3), so does each of the three pillow operators. This means that the scaling dimensions of the pillow operators are unaffected by the interactions, i.e. they vanish. The gauging of the $S O(N)^{3}$ symmetry sets the charges (5.5) to zero, so the pillow operators do not appear in the gauged model.

Using the equations of motion we see that the operator $O_{\text {tetra }}$ is related by the equation of motion to the operator $O_{2}^{1}$ :

$$
O_{\text {tetra }}=\psi^{a b c}\left(\psi^{3}\right)^{a b c} \propto \psi^{a b c} \partial_{t} \psi^{a b c} .
$$

If we iterate the use of the equation of motion, then all derivatives in an operator may be traded for extra $\psi$-fields. Thus, a complete basis of operators may be constructed by multiplying some number $2 k$ of $\psi$-fields and contracting all indices. In this approach, there is a unique operator with $k=2(m+1)$, which is equal to the Regge trajectory operator $O_{2}^{2 m+1}$. For $m=0$ this operator is $O_{\text {tetra }}$, which is proportional to the Hamiltonian. 
All the six-particle operators vanish by the Fermi statistics, but there is a number of eightparticle ones. We will exhibit only the ones not containing bubble insertions. Having two vertices connected by a double line corresponds to insertion of an $S O(N)$ charge which vanishes in the gauged model. For this reason we will omit such operators and list only those where there are no double lines. In [54] it was shown that there are 17 inequivalent operators; see figure 25. For example, the three operators shown in the first column of the figure 25 are

$$
\begin{aligned}
& O_{1}=\psi^{a_{1} b_{1} c_{1}} \psi^{a_{1} b_{2} c_{2}} \psi^{a_{2} b_{2} c_{1}} \psi^{a_{2} b_{4} c_{4}} \psi^{a_{3} b_{3} c_{2}} \psi^{a_{3} b_{1} c_{3}} \psi^{a_{4} b_{4} c_{3}} \psi^{a_{4} b_{3} c_{4}}=\partial_{t} \psi^{a_{1} b_{1} c_{1}} \partial_{t} \psi^{a_{1} b_{2} c_{2}} \psi^{a_{2} b_{1} c_{2}} \psi^{a_{2} b_{2} c_{1}}, \\
& O_{2}=\psi^{a_{1} b_{1} c_{1}} \psi^{a_{1} b_{2} c_{2}} \psi^{a_{2} b_{2} c_{1}} \psi^{a_{2} b_{3} c_{3}} \psi^{a_{3} b_{3} c_{2}} \psi^{a_{3} b_{4} c_{4}} \psi^{a_{4} b_{4} c_{3}} \psi^{a_{4} b_{1} c_{4}}=\partial_{t} \psi^{a_{1} b_{1} c_{1}} \psi^{a_{1} b_{2} c_{2}} \partial_{t} \psi^{a_{2} b_{1} c_{2}} \psi^{a_{2} b_{2} c_{1}}, \\
& O_{3}=\psi^{a_{1} b_{1} c_{1}} \psi^{a_{1} b_{2} c_{2}} \psi^{a_{2} b_{2} c_{1}} \psi^{a_{2} b_{3} c_{3}} \psi^{a_{3} b_{1} c_{3}} \psi^{a_{3} b_{4} c_{4}} \psi^{a_{4} b_{3} c_{4}} \psi^{a_{4} b_{4} c_{2}}=\partial_{t} \psi^{a_{1} b_{1} c_{1}} \psi^{a_{1} b_{2} c_{2}} \psi^{a_{2} b_{1} c_{2}} \partial_{t} \psi^{a_{2} b_{2} c_{1}}
\end{aligned}
$$

It follows that

$$
O_{1}+O_{2}+O_{3} \sim \partial_{t} \psi^{a b c} \partial_{t}^{2} \psi^{a b c},
$$

which up to a total derivative equals the Regge trajectory operator $O_{2}^{3}$.
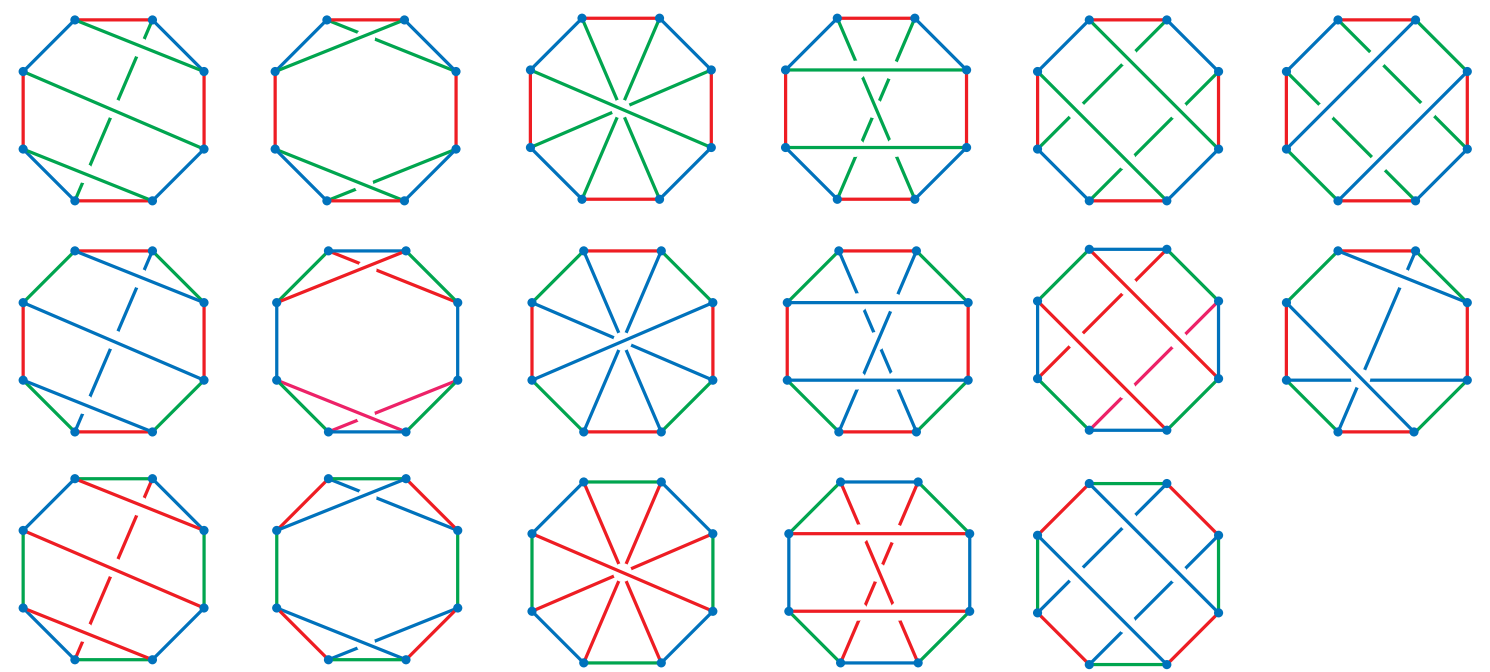

Figure 25: All eight-particle operators in the gauged fermionic model.

The higher bubble-free single-sum operators were counted in [54]: there are 24 ten-particle ones, 617 twelve-particle ones, 4887 fourteen-particle ones, and 82466 sixteen-particle ones. In the bosonic theory, the single-sum operators correspond to connected Feynman diagrams in the theory with three scalar fields and $\phi_{1} \phi_{2} \phi_{3}$ interaction. The number of such diagrams with $2 k$ vertices grows as $k$ !, and so does the number of invariant single-sum operators in the bosonic theory [114119]. In the fermionic theory some operators vanish due to the Fermi statistics, but the factorial growth remains: in [54] it was shown that the number of $(2 k)$-particle $S O(N)^{3}$ invariant single-sum operators grows like $\sim k ! 2^{k}$. This is much faster than the exponential growth found in string theory. It implies that the Hagedorn temperature vanishes as $1 / \log N$ and suggests that the dual description of the tensor models lies "beyond string theory." Perhaps it is related to M-theory, as suggested by the $N^{3}$ growth of the number of degrees of freedom on the M5-branes [47] which may correspond to the M2-branes of trinion topology. 


\section{Tensor Models for Complex Fermions}

In this section we study two different quantum mechanical models of a complex 3-tensor $\psi^{a b c}$. One possibility is the model

$$
S=\int d t\left(i \bar{\psi}^{a b c} \partial_{t} \psi^{a b c}-\frac{1}{2} g \bar{\psi}^{a_{1} b_{2} c_{2}} \bar{\psi}^{a_{2} b_{2} c_{1}} \psi^{a_{1} b_{1} c_{1}} \psi^{a_{2} b_{1} c_{2}}\right) .
$$

Its symmetry is $S U(N) \times O(N) \times S U(N) \times U(1)$, where the $S U(N)$ groups act on the first and third indices. ${ }^{5}$ The $U(1)$ acts by a phase rotation, $\psi^{a b c} \rightarrow e^{i \alpha} \psi^{a b c}$, and the corresponding conserved charge is

$$
Q=\frac{1}{2}\left[\bar{\psi}^{a b c}, \psi^{a b c}\right] .
$$

The model (6.1) is the tensor counterpart of the variant of SYK model where the real fermions are replaced by the complex ones [55,56, 121-125].

Let us study the conformal primary operators of the form

$$
\mathscr{O}_{2}^{n}=\bar{\psi}^{a b c}\left(\partial_{t}^{n} \psi\right)^{a b c} \quad n=0,1, \ldots
$$

up to total derivatives (there is a variety of operators made out of the higher powers of the fermionic fields, and some of them are equivalent to (6.3) via the equations of motion). As established in $[30,37,120]$, the large $N$ limit of the complex model (6.1) is once again given by the melon diagrams (the arguments are easier than in section 4 since each index loop passes through an even number of vertices). Let us briefly discuss summing over melonic graphs in the model (6.1) at large $N$. The two-point function has the structure

$$
\left\langle T\left(\bar{\psi}^{a b c}(t) \psi^{a^{\prime} b^{\prime} c^{\prime}}(0)\right)\right\rangle=\delta^{a a^{\prime}} \delta^{b b^{\prime}} \delta^{c c^{\prime}} G(t),
$$

and $G(t)=-G(-t)$. We find the same Schwinger-Dyson equation as (5.20); its solution is again (6.14) indicating that the fermion scaling dimension is $\Delta=1 / 4$. Now we need to study the 4point function $\left\langle\bar{\psi}^{a_{1} b_{1} c_{1}}\left(t_{1}\right) \psi^{a_{1} b_{1} c_{1}}\left(t_{2}\right) \bar{\psi}^{a_{2} b_{2} c_{2}}\left(t_{3}\right) \psi^{a_{2} b_{2} c_{2}}\left(t_{4}\right)\right\rangle$. It leads to the same integral eigenvalue equation as (5.28), but with kernel

$$
K\left(t_{1}, t_{2} ; t_{3}, t_{4}\right)=-g^{2} N^{3}\left(2 G\left(t_{13}\right) G\left(t_{24}\right) G\left(t_{34}\right)^{2}-G\left(t_{14}\right) G\left(t_{23}\right) G\left(t_{34}\right)^{2}\right) .
$$

Now it is possible to have not only the antisymmetric eigenfunctions as in (5.29), but also the symmetric ones

$$
v_{2 n}\left(t_{0}, t_{1}, t_{2}\right)=\left\langle\mathscr{O}_{2}^{2 n}\left(t_{0}\right) \psi^{a b c}\left(t_{1}\right) \bar{\psi}^{a b c}\left(t_{2}\right)\right\rangle=\frac{c_{2 n} \operatorname{sgn}\left(t_{0}-t_{1}\right) \operatorname{sgn}\left(t_{0}-t_{2}\right)}{\left|t_{0}-t_{1}\right|^{h}\left|t_{0}-t_{2}\right|^{h}\left|t_{1}-t_{2}\right|^{1 / 2-h}} .
$$

This can be justified by noticing that the three point function now is $\left\langle\mathscr{O}_{2}^{n}\left(t_{0}\right) \psi^{a b c}\left(t_{1}\right) \bar{\psi}^{a b c}\left(t_{2}\right)\right\rangle$. We see that for odd $n$ it is antisymmetric under $t_{1} \leftrightarrow t_{2}$, while for even $n$ it is symmetric.

Substituting ansatz (6.6) into the integral equation, and using the integrals (5.30), we find

$$
g_{\text {sym }}(h)=-\frac{1}{2} \frac{\tan \left(\frac{\pi}{2}\left(h+\frac{1}{2}\right)\right)}{h-1 / 2} .
$$

\footnotetext{
${ }^{5}$ Bosonic models with this symmetry were previously studied in $d=0[30,37,120]$.
} 
The scaling dimensions of the operators $\mathscr{O}_{2}^{n}$ with even $n$ are given by the solutions of $g_{\text {sym }}(h)=1$. The first eigenvalue is $h=1$, corresponding to the conserved $U(1)$ charge. The additional values are $h \approx 2.65,4.58,6.55,8.54$ corresponding to the operators with $n=2,4,6,8$ respectively. For large $n$ the scaling dimensions approach $n+\frac{1}{2}$ as expected. The numerical results are in good agreement with the asymptotic formula [98]

$$
h_{n}=n+\frac{1}{2}+\frac{1}{\pi n}+\mathscr{O}\left(n^{-3}\right)
$$

for $n>2$. For $\mathscr{O}_{2}^{n}$ with odd $n$ the spectrum is the same as for the two-particle operators (5.11) in the Majorana model with $O(N)^{3}$ symmetry. The plot of the graphical solution for the scaling dimensions is shown in figure 26, with odd $n$ in orange and even $n$ in black.

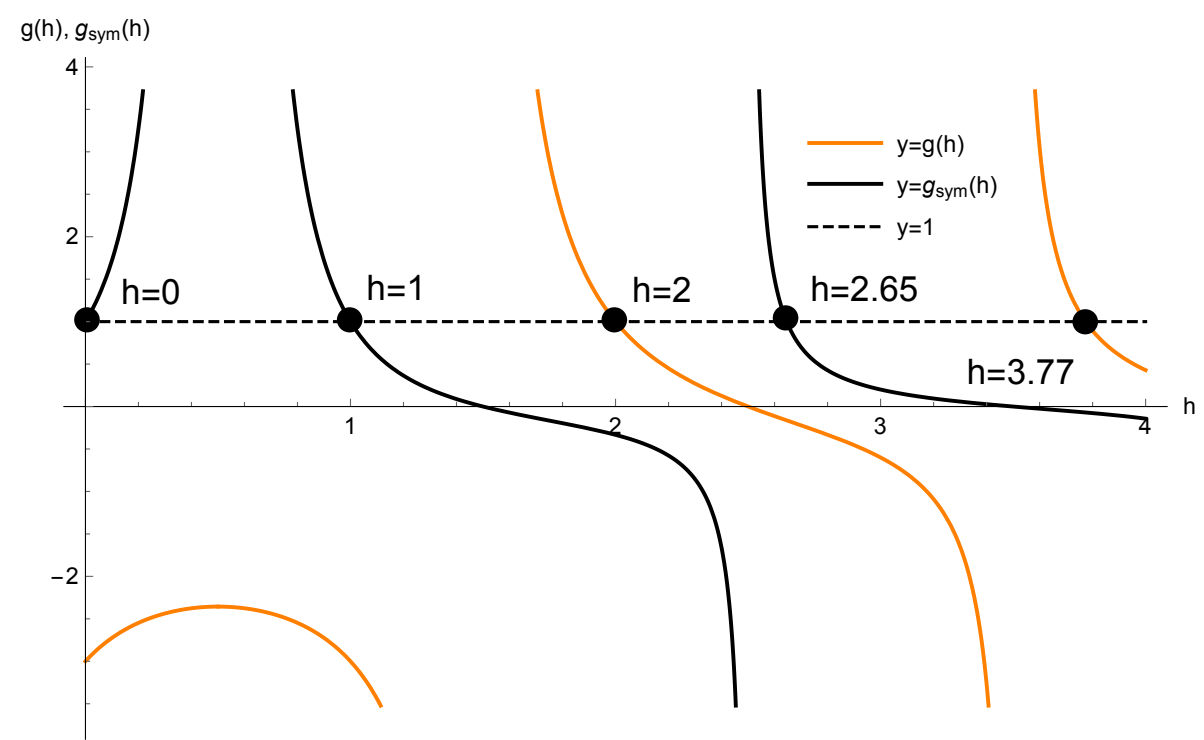

Figure 26: Graphical solution of the equations $g_{\text {antisym }}(h)=1$ (in orange) and $g_{\text {sym }}(h)=1$ (in black).

\subsection{Bipartite complex tensor model}

Another possible complex model is [34]:

$$
S=\int d t\left(i \bar{\psi}^{a b c} \partial_{t} \psi^{a b c}+\frac{1}{4} g \psi^{a_{1} b_{1} c_{1}} \psi^{a_{1} b_{2} c_{2}} \psi^{a_{2} b_{1} c_{2}} \psi^{a_{2} b_{2} c_{1}}+\frac{1}{4} \bar{g} \bar{\psi}^{a_{1} b_{1} c_{1}} \bar{\psi}^{a_{1} b_{2} c_{2}} \bar{\psi}^{a_{2} b_{1} c_{2}} \bar{\psi}^{a_{2} b_{2} c_{1}}\right) \text {. }
$$

It is a special case of a more general model with $O(N)^{3}$ symmetry, which was studied in [126]. In the Feynman graph expansion for the model (6.9) we denote the $\bar{\psi}^{4}$ and $\psi^{4}$ vertices by white and black dots, respectively (see figure 27). Each Feynman graph necessarily has equal number of black and white dots; therefore, it is called a bipartite graph (a similar bipartite model for multiple complex fermions was studied in [127]).

In the model (6.9) there is no $U(1)$ phase rotation symmetry; the interaction breaks it to $\mathbb{Z}_{4}$. For real $g$ there is an additional symmetry

$$
\mathbb{Z}_{2}: \quad \psi \rightarrow \bar{\psi} .
$$



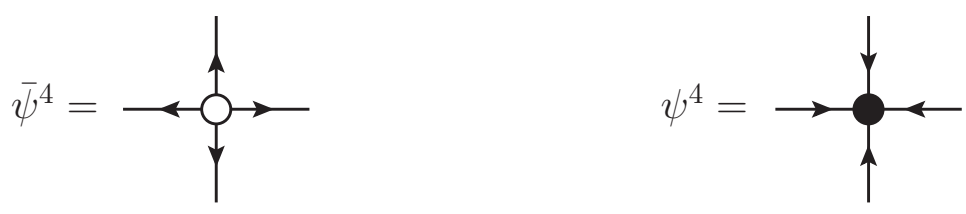

Figure 27: Vertices in the bipartite complex model

If we decompose a complex tensor into two real ones, $\psi^{a b c}=\psi_{1}^{a b c}+i \psi_{2}^{a b c}$, then this $\mathbb{Z}_{2}$ acts by $\psi_{2}^{a b c} \rightarrow-\psi_{2}^{a b c}$.

In the large $N$ limit this model is dominated by the bipartite melonic diagrams. The two-point function

$$
\left\langle T\left(\bar{\psi}^{a b c}(t) \psi^{a^{\prime} b^{\prime} c^{\prime}}(0)\right)\right\rangle=\delta^{a a^{\prime}} \delta^{b b^{\prime}} \delta^{c c^{\prime}} G(t)
$$

satisfies the Schwinger-Dyson equation

$$
G\left(t_{1}-t_{2}\right)=G_{0}\left(t_{1}-t_{2}\right)-g \bar{g} N^{3} \int d t d t^{\prime} G_{0}\left(t_{1}-t\right) G\left(t^{\prime}-t\right)^{3} G\left(t^{\prime}-t_{2}\right),
$$

which is graphically depicted in figure 28 .

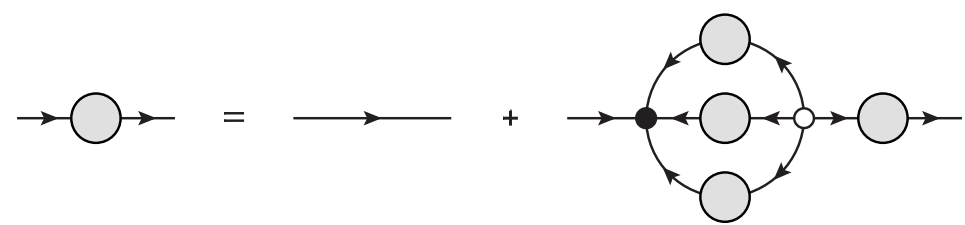

Figure 28: The graphical representation of the Schwinger-Dyson equation for the two-point function in the bipartite model.

Since there is no chemical potential, we have $G(-t)=-G(t)$. Therefore, the Schwinger-Dyson equation may be written as

$$
G\left(t_{1}-t_{2}\right)=G_{0}\left(t_{1}-t_{2}\right)+g \bar{g} N^{3} \int d t d t^{\prime} G_{0}\left(t_{1}-t\right) G\left(t-t^{\prime}\right)^{3} G\left(t^{\prime}-t_{2}\right),
$$

which is the same as in the $O(N)^{3}$ model with a real 3-tensor [34]. The solution is given by the formula

$$
G\left(t_{1}-t_{2}\right)=-\left(\frac{1}{4 \pi \lambda^{2}}\right)^{1 / 4} \frac{\operatorname{sgn}\left(t_{1}-t_{2}\right)}{\left|t_{1}-t_{2}\right|^{1 / 2}}
$$

where $\lambda^{2}=|g|^{2} N^{3}$ is kept fixed. This indicates that the fermion scaling dimension is $\Delta=1 / 4$.

Let us study the fermion bilinear operators

$$
O_{n}=\bar{\psi}^{a b c} \partial_{t}^{n} \psi^{a b c}
$$

For odd $n$ they are even under the $\mathbb{Z}_{2}$ symmetry (6.10), while for even $n$ they are odd under the $\mathbb{Z}_{2}$. This is not hard to see using the real tensors $\psi_{i}, i=1,2$. As is well-known for the model with a 
real tensor [34], or equivalently for the SYK model [98], for even $n$ there are no primary operators of the form $\psi_{i}^{a b c} \partial_{t}^{n} \psi_{i}^{a b c}$. Therefore, the primary operators for even $n$ are the $\mathbb{Z}_{2}$-odd operators $\psi_{1}^{a b c} \partial_{t}^{n} \psi_{2}^{a b c}$.

To compute the scaling dimensions of $O_{n}$ we consider a three point function

$$
v_{n}\left(t_{0}, t_{1}, t_{2}\right)=\left\langle O_{n}\left(t_{0}\right) \psi^{a b c}\left(t_{1}\right) \bar{\psi}^{a b c}\left(t_{2}\right)\right\rangle .
$$

Then one can derive the Schwinger-Dyson equation for the three point function in the IR region [99]

$$
v_{n}\left(t_{0}, t_{1}, t_{2}\right)=\int d t_{3} d t_{4} K\left(t_{1}, t_{2}, t_{3}, t_{4}\right) v_{n}\left(t_{0}, t_{3}, t_{4}\right),
$$

where the kernel is

$$
K\left(t_{1}, t_{2}, t_{3}, t_{4}\right)=3 \lambda^{2} G\left(t_{14}\right) G\left(t_{23}\right) G\left(t_{34}\right)^{2} .
$$

The Schwinger-Dyson equation (6.17) represented graphically in figure 29, where we have already dropped the bare term, which is irrelevant in the IR region.
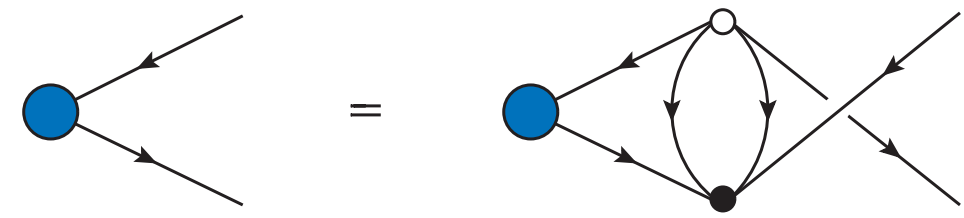

Figure 29: The graphical representation of the Schwinger-Dyson equation for the three-point function.

For odd $n$ the three point function is antisymmetric $v\left(t_{0}, t_{1}, t_{2}\right)=-v\left(t_{0}, t_{2}, t_{1}\right)$ and is given by the general formula

$$
v_{n}\left(t_{0}, t_{1}, t_{2}\right)=\frac{c_{n} \operatorname{sgn}\left(t_{1}-t_{2}\right)}{\left|t_{01}\right|^{h}\left|t_{02}\right|^{h}\left|t_{12}\right|^{\frac{1}{2}-h}},
$$

where $h$ is the anomalous dimension of the operator $O_{n}$ and $c_{n}$ is the structure constant ${ }^{6}$. For arbitrary $h$ the three point function (6.19) satisfies the equation

$$
v_{n}\left(t_{0}, t_{1}, t_{2}\right)=g_{A}(h) \int d t_{3} d t_{4} K\left(t_{1}, t_{2}, t_{3}, t_{4}\right) v_{n}\left(t_{0}, t_{3}, t_{4}\right),
$$

where

$$
g_{A}(h)=-\frac{3}{2} \frac{\tan \left(\frac{\pi}{2}\left(h-\frac{1}{2}\right)\right)}{h-1 / 2} .
$$

For even $n$ the three point function is symmetric $v\left(t_{0}, t_{1}, t_{2}\right)=v\left(t_{0}, t_{2}, t_{1}\right)$ and is given by the general formula

$$
v_{n}\left(t_{0}, t_{1}, t_{2}\right)=\frac{c_{n} \operatorname{sgn}\left(t_{0}-t_{1}\right) \operatorname{sgn}\left(t_{0}-t_{2}\right)}{\left|t_{01}\right|^{h}\left|t_{02}\right|^{h}\left|t_{12}\right|^{\frac{1}{2}-h}}
$$

\footnotetext{
${ }^{6}$ This method of finding anomalous dimension does not determine the structure constants $c_{n}$. One can find them by computing the whole four point function [98].
} 
where $h$ is the anomalous dimension of the operator $O_{n}$ and $c_{n}$ is the structure constant. For arbitrary $h$ the three point function (6.22) satisfies the equation

$$
v_{n}\left(t_{0}, t_{1}, t_{2}\right)=g_{S}(h) \int d t_{3} d t_{4} K\left(t_{1}, t_{2}, t_{3}, t_{4}\right) v_{n}\left(t_{0}, t_{3}, t_{4}\right),
$$

where

$$
g_{S}(h)=\frac{3}{2} \frac{\tan \left(\frac{\pi}{2}\left(h+\frac{1}{2}\right)\right)}{h-1 / 2} .
$$

Now to find anomalous dimensions of the operators $O_{n}$ we have to find solutions to the equations

$$
g_{S}(h)=1, \quad g_{A}(h)=1 .
$$

We find a series of real solutions, which approach $h_{n} \rightarrow n+\frac{1}{2}$ at large $n$. The first few values are $h=2,2,3.77,4.26,5.68,6.34$ corresponding to $n=1,2,3,4,5,6$, respectively. The plot is represented in figure 30. An interesting feature of this model is that both operators $O_{1}=\bar{\psi}^{a b c} \partial_{t} \psi^{a b c}$ and $O_{2}=\bar{\psi}^{a b c} \partial_{t}^{2} \psi^{a b c}$ have the same exact eigenvalue equal to $h=2$. For $n=1$ this eigenvalue corresponds to a normalizable state, but presumably this is not the case for $n=2$.

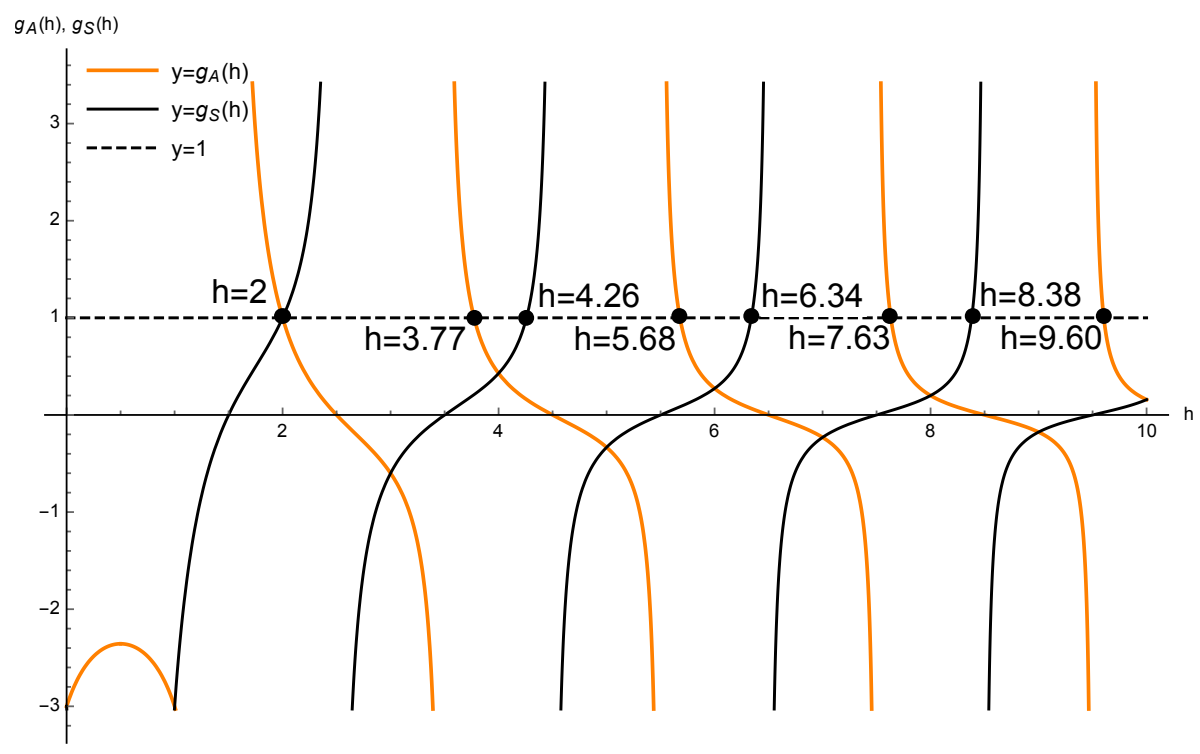

Figure 30: Graphical solution of the equations $g_{S}(h)=1$ and $g_{A}(h)=1$.

The Schwinger -Dyson solution of the bipartite model has a new feature compared to the tensor models discussed in previous sections. For $n=0$, which corresponds to the operator $O=\bar{\psi}^{a b c} \psi^{a b c}$, the solution of the equation $g_{S}(h)=1$ is complex:

$$
h \approx \frac{1}{2}+1.5251 i \text {. }
$$

This signals a likely instability of this nearly-conformal model. From the point of view of the dual theory in $A d S_{2}$, the mass-squared of a a scalar field dual to an operator of dimension $h$ is

$$
m^{2}=h(h-1) .
$$

Therefore, a complex $h$ of the form $\frac{1}{2}+i \alpha$ corresponds to $m^{2}=m_{\mathrm{BF}}^{2}-\alpha^{2}$. This is below the Breitenlohner-Freedman [128] stability bound $m_{\mathrm{BF}}^{2}=-\frac{1}{4}$, thus causing an instability. 


\section{Bosonic Tensor Models}

It is of obvious interest to try extending the derivations above to quantum theories of bosonic tensors. It turns out that they frequently contain complex scaling dimensions, such as those noted in the previous section. In [34,89] an $O(N)^{3}$ invariant theory of the scalar fields $\phi^{a b c}$ was explored. When endowed with a quartic "tetrahedral" potential $[32,34]$

$$
V=\frac{g}{4 !} \phi^{a_{1} b_{1} c_{1}} \phi^{a_{1} b_{2} c_{2}} \phi^{a_{2} b_{1} c_{2}} \phi^{a_{2} b_{2} c_{1}},
$$

this theory is super-renormalizable in $d<4$ and is formally solvable using the Schwinger-Dyson equations. However, this model has some instabilities. One problem is that the tetrahedral potential is not positive definite. Even if we ignore this and consider the large $N$ limit formally, we find that in $d<4$ the $O(N)^{3}$ invariant operator $\phi^{a b c} \phi^{a b c}$ has a complex dimension of the form $\frac{d}{2}+i \alpha(d)$, which leads to instabilitiy of the resulting large $N$ CFT. ${ }^{7}$ From the dual AdS point of view, such a complex dimension corresponds to a scalar field whose $m^{2}$ is below the Breitenlohner-Freedman stability bound $[128,133]$. The origin of the complex dimensions was elucidated using perturbation theory in $4-\varepsilon$ dimensions: the fixed point was found to be at complex values of the couplings for the additional $O(N)^{3}$ invariant operators required by the renormalizability [34,89]. In [89] a $O(N)^{5}$ symmetric theory for tensor $\phi^{a b c d e}$ and sextic interactions was also considered. It was found that the dimension of $\phi^{a b c d e} \phi^{a b c d e}$ is real in the narrow range $d_{\text {crit }}<d<3$, where $d_{\text {crit }} \approx 2.97$. However, the scalar potential of this theory is again unstable, so the theory may be defined only formally. In spite of these problems, some interesting formal results on melonic scalar theories of this type were found recently [134].

In [35], the search was continued for stable non-supersymmetric large $N$ scalar theories with multiple $O(N)$ symmetry groups. The specific model studied was the $O(N)^{3}$ symmetric theory of scalar fields $\phi^{a b c}$ with a sextic interaction, whose Euclidean action in $d$ dimensions is

$$
S=\int d^{d} x\left(\frac{1}{2}\left(\partial_{\mu} \phi^{a b c}\right)^{2}+\frac{g_{1}}{6 !} \phi^{a_{1} b_{1} c_{1}} \phi^{a_{1} b_{2} c_{2}} \phi^{a_{2} b_{1} c_{2}} \phi^{a_{3} b_{3} c_{1}} \phi^{a_{3} b_{2} c_{3}} \phi^{a_{2} b_{3} c_{3}}\right) .
$$

This theory is super-renormalizable in $d<3$. When fields $\phi^{a b c}$ are represented by vertices and index contractions by edges, this interaction term looks like a prism [34]; it is the leftmost diagram in figure 31. In the large $N$ limit, $g_{1} N^{3}$ is held fixed [35]. We may call this theory "prismatic" (note that the Feynman diagrams are not the same as the melonic diagrams which appear in the sextic theory of [89] with the $O(N)^{5}$ symmetry). Unlike with the quartic interaction of tetrahedral topology $[34,89]$, the action $(7.2)$ is positive for $g_{1}>0$. In fact, it is the bosonic part of the action of the supersymmetric theory with two supercharges and tetrahedral superpotential [34], $W \sim \Phi^{a_{1} b_{1} c_{1}} \Phi^{a_{1} b_{2} c_{2}} \Phi^{a_{2} b_{1} c_{2}} \Phi^{a_{2} b_{2} c_{1}}$.

The theory (7.2) may be viewed as a tensor counterpart of the bosonic theory with random couplings, which was introduced in section 6.2 of [135]. Since both theories are dominated by the same class of diagrams in the large $N$ limit, they have the same Schwinger-Dyson equations for the 2-point and 4-point functions. For $1.68<d<2.81$ there is a scalar bilinear operator with a complex dimension of the form $\frac{d}{2}+i \alpha(d)$, but outside of this range there is no such complex

\footnotetext{
${ }^{7}$ Complex scaling dimensions of this form appear in various other large $N$ theories; see, for example, [129-132].
} 
scaling dimension [35]. This raises the possibility of a purely bosonic near-conformal quantum mechanical theory. ${ }^{8}$

One can also use the renormalized perturbation theory to develop the $3-\varepsilon$ expansion. To carry out the beta function calculation at finite $N$ we need to include all the $O(N)^{3}$ invariant sextic terms in the action. The 11 such single-sum terms are shown diagrammatically in figure 5 of [54]. It is convenient to impose the additional constraint that the action is invariant under the permutation group $S_{3}$ which acts on the three $O(N)$ symmetry groups. The most essential for achieving the large $N$ limit is the "prism" term (7.2); it is positive definite and symmetric under the interchanges of the three $O(N)$ groups. The 8 needed operators and associated couplings are exhibited in figure 31 , with the prism operator shown on the left. The coupled two-loop beta functions, calculated in [35], have a "prismatic" fixed point for $N>53$, where all the coupling constants are real. As $N$ is increased, $g_{1} \sim N^{-3}$ at this fixed point, while the other 7 couplings scale to zero faster. The resulting $3-\varepsilon$ expansions are, in the large $N$ limit, in agreement with the results from the Schwinger-Dyson equations used to solve the prismatic large $N$ theory. This constitutes an explicit perturbative check of the large $N$ solution of the prismatic theory [35].

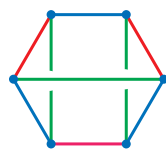

$g_{1}$

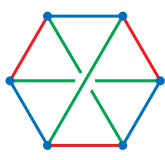

$g_{2}$

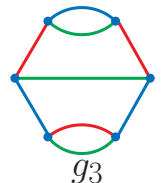

$g_{3}$

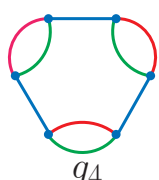

$g_{4}$

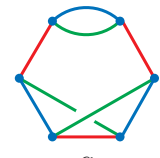

$g_{5}$

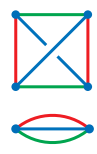

$g_{6}$

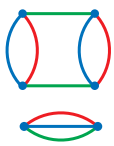

$g_{7}$

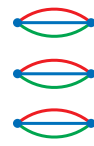

$g_{8}$

Figure 31: Diagrammatic representation of the eight possible $O(N)^{3}$ invariant sextic interaction terms.

\section{Acknowledgments}

These notes are an expanded version of the lectures presented by IRK at the TASI 2017 summer school in June 2017 in Boulder, Colorado, and at the Abdus Salam ICTP 2018 Spring School in March 2018 in Trieste, Italy. IRK is grateful to the TASI 2017 co-organizers Mirjam Cvetic, Tom DeGrand and Oliver DeWolfe for creating a wonderful environment at the school. He is also grateful to the organizers of the 2018 ICTP Spring School, especially Atish Dabholkar, for the invitation and hospitality. Many thanks to the students at both school for the many good questions and useful discussions. We thank our collaborators K. Bulycheva, S. Giombi, A. Milekhin, K. Pakrouski, and S. Prakash, with whom some of the results reviewed here were obtained. We are grateful to D. Gross, R. Gurau, J. Maldacena, V. Rosenhaus, D. Stanford, and E. Witten, for illuminating discussions. The work of IRK and FP was supported in part by the US NSF under Grant No. PHY-1620059. The work of GT was supported by the MURI grant W911NF-14-1-0003 from ARO and by DOE grant de-sc0007870.

\footnotetext{
${ }^{8}$ Another quantum mechanical theory containing bosonic tensors, where the supersymmetry is broken spontaneously, was recently studied in [136].
} 


\section{References}

[1] H. E. Stanley, Spherical model as the limit of infinite spin dimensionality, Phys. Rev. 176 (1968) 718.

[2] K. G. Wilson and J. B. Kogut, The Renormalization group and the epsilon expansion, Phys. Rept. 12 (1974) 75 .

[3] M. Moshe and J. Zinn-Justin, Quantum field theory in the large N limit: A Review, Phys. Rept. 385 (2003) 69 [hep-th/0306133].

[4] G. 't Hooft, A Planar Diagram Theory for Strong Interactions, Nucl. Phys. B72 (1974) 461.

[5] G. Veneziano, Some Aspects of a Unified Approach to Gauge, Dual and Gribov Theories, Nucl. Phys. B117 (1976) 519.

[6] J. M. Maldacena, The Large N limit of superconformal field theories and supergravity, Int. J. Theor. Phys. 38 (1999) 1113 [hep-th/9711200].

[7] S. S. Gubser, I. R. Klebanov and A. M. Polyakov, Gauge theory correlators from noncritical string theory, Phys. Lett. B428 (1998) 105 [hep-th/9802109].

[8] E. Witten, Anti-de Sitter space and holography, Adv. Theor. Math. Phys. 2 (1998) 253 [hep-th/9802150].

[9] O. DeWolfe, TASI Lectures on Applications of Gauge/Gravity Duality, in Theoretical Advanced Study Institute in Elementary Particle Physics: Physics at the Fundamental Frontier (TASI 2017) Boulder, CO, USA, June 5-30, 2017, 2018, 1802.08267.

[10] J. Erdmenger, Introduction to Gauge/Gravity Duality (TASI Lectures 2017), PoS TASI2017 (2017) $001[1807.09872]$.

[11] D. Harlow, TASI Lectures on the Emergence of the Bulk in AdS/CFT, 1802.01040.

[12] I. R. Klebanov, TASI lectures: Introduction to the AdS / CFT correspondence, in Strings, branes and gravity. Proceedings, Theoretical Advanced Study Institute, TASI'99, Boulder, USA, May 31-June 25, 1999, pp. 615-650, 2000, hep-th/0009139, DOI.

[13] E. D'Hoker and D. Z. Freedman, Supersymmetric gauge theories and the AdS / CFT correspondence, in Strings, Branes and Extra Dimensions: TASI 2001: Proceedings, pp. 3-158, 2002, hep-th/0201253.

[14] J. M. Maldacena, TASI 2003 lectures on AdS / CFT, in Progress in string theory. Proceedings, Summer School, TASI 2003, Boulder, USA, June 2-27, 2003, pp. 155-203, 2003, hep-th/0309246.

[15] O. Aharony, S. S. Gubser, J. M. Maldacena, H. Ooguri and Y. Oz, Large N field theories, string theory and gravity, Phys. Rept. 323 (2000) 183 [hep-th/9905111].

[16] E. Brezin, C. Itzykson, G. Parisi and J. B. Zuber, Planar Diagrams, Commun. Math. Phys. 59 (1978) 35.

[17] D. J. Gross and A. A. Migdal, Nonperturbative Two-Dimensional Quantum Gravity, Phys. Rev. Lett. 64 (1990) 127.

[18] E. Brezin and V. A. Kazakov, Exactly Solvable Field Theories of Closed Strings, Phys. Lett. B236 (1990) 144.

[19] M. R. Douglas and S. H. Shenker, Strings in Less Than One-Dimension, Nucl. Phys. B335 (1990) 635. 
[20] P. H. Ginsparg and G. W. Moore, Lectures on 2-D gravity and 2-D string theory, in Proceedings, Theoretical Advanced Study Institute (TASI 92): From Black Holes and Strings to Particles: Boulder, USA, June 1-26, 1992.

[21] I. R. Klebanov, String theory in two-dimensions, in Spring School on String Theory and Quantum Gravity (to be followed by Workshop) Trieste, Italy, April 15-23, 1991, pp. 30-101, 1991, hep-th/9108019.

[22] A. M. Polyakov, Quantum Geometry of Bosonic Strings, Phys. Lett. B103 (1981) 207.

[23] J. Ambjorn, B. Durhuus and T. Jonsson, Three-dimensional simplicial quantum gravity and generalized matrix models, Mod. Phys. Lett. A6 (1991) 1133.

[24] N. Sasakura, Tensor model for gravity and orientability of manifold, Mod. Phys. Lett. A6 (1991) 2613.

[25] M. Gross, Tensor models and simplicial quantum gravity in $>2-D$, Nucl. Phys. Proc. Suppl. 25A (1992) 144.

[26] R. Gurau, Colored Group Field Theory, Commun. Math. Phys. 304 (2011) 69 [0907. 2582].

[27] R. Gurau and V. Rivasseau, The 1/N expansion of colored tensor models in arbitrary dimension, Europhys. Lett. 95 (2011) 50004 [1101.4182].

[28] R. Gurau, The complete 1/N expansion of colored tensor models in arbitrary dimension, Annales Henri Poincare 13 (2012) 399 [1102. 5759].

[29] V. Bonzom, R. Gurau, A. Riello and V. Rivasseau, Critical behavior of colored tensor models in the large N limit, Nucl. Phys. B853 (2011) 174 [1105.3122].

[30] A. Tanasa, Multi-orientable Group Field Theory, J. Phys. A45 (2012) 165401 [1109. 0694 ].

[31] V. Bonzom, R. Gurau and V. Rivasseau, Random tensor models in the large N limit: Uncoloring the colored tensor models, Phys. Rev. D85 (2012) 084037 [1202.3637].

[32] S. Carrozza and A. Tanasa, $O(N)$ Random Tensor Models, Lett. Math. Phys. 106 (2016) 1531 [1512.06718].

[33] E. Witten, An SYK-Like Model Without Disorder, 1610.09758.

[34] I. R. Klebanov and G. Tarnopolsky, Uncolored random tensors, melon diagrams, and the Sachdev-Ye-Kitaev models, Phys. Rev. D95 (2017) 046004 [1611. 08915 ].

[35] S. Giombi, I. R. Klebanov, F. Popov, S. Prakash and G. Tarnopolsky, Prismatic Large N Models for Bosonic Tensors, 1808.04344.

[36] R. Gurau and J. P. Ryan, Colored Tensor Models - a review, SIGMA 8 (2012) 020 [1109.4812].

[37] A. Tanasa, The Multi-Orientable Random Tensor Model, a Review, SIGMA 12 (2016) 056 [1512.02087].

[38] N. Delporte and V. Rivasseau, The Tensor Track V: Holographic Tensors, 2018, 1804.11101.

[39] S. Sachdev and J. Ye, Gapless spin fluid ground state in a random, quantum Heisenberg magnet, Phys. Rev. Lett. 70 (1993) 3339 [cond-mat/9212030].

[40] O. Parcollet and A. Georges, Non-Fermi-liquid regime of a doped Mott insulator, Physical Review B 59 (1999) 5341 [cond-mat/9806119]. 
[41] A. Georges, O. Parcollet and S. Sachdev, Mean Field Theory of a Quantum Heisenberg Spin Glass, Physical Review Letters 85 (2000) 840 [cond-mat/9909239].

[42] A. Kitaev, A simple model of quantum holography, .

[43] A. Kitaev and S. J. Suh, The soft mode in the Sachdev-Ye-Kitaev model and its gravity dual, JHEP 05 (2018) 183 [1711.08467].

[44] G. Sarosi, AdS 2 holography and the SYK model, PoS Modave2017 (2018) 001 [1711. 08482 ].

[45] V. Rosenhaus, An introduction to the SYK model, 1807.03334.

[46] O. Aharony, O. Bergman, D. L. Jafferis and J. Maldacena, N=6 superconformal Chern-Simons-matter theories, M2-branes and their gravity duals, JHEP 10 (2008) 091 [0806.1218].

[47] I. R. Klebanov and A. A. Tseytlin, Entropy of near extremal black p-branes, Nucl. Phys. B475 (1996) 164 [hep-th/9604089].

[48] N. Drukker, M. Marino and P. Putrov, From weak to strong coupling in ABJM theory, Commun. Math. Phys. 306 (2011) 511 [1007.3837].

[49] C. P. Herzog, I. R. Klebanov, S. S. Pufu and T. Tesileanu, Multi-Matrix Models and Tri-Sasaki Einstein Spaces, Phys. Rev. D83 (2011) 046001 [1011. 5487 ].

[50] M. Marino and P. Putrov, ABJM theory as a Fermi gas, J. Stat. Mech. 1203 (2012) P03001 [1110.4066].

[51] M. Marino, Localization at large N in ChernâĂŞSimons-matter theories, J. Phys. A50 (2017) 443007 [1608.02959].

[52] S. S. Pufu, The F-Theorem and F-Maximization, J. Phys. A50 (2017) 443008 [1608 . 02960 ].

[53] D. L. Jafferis, I. R. Klebanov, S. S. Pufu and B. R. Safdi, Towards the F-Theorem: N=2 Field Theories on the Three-Sphere, JHEP 06 (2011) 102 [1103.1181].

[54] K. Bulycheva, I. R. Klebanov, A. Milekhin and G. Tarnopolsky, Spectra of Operators in Large N Tensor Models, Phys. Rev. D97 (2018) 026016 [1707.09347].

[55] S. Sachdev, Bekenstein-Hawking Entropy and Strange Metals, Phys. Rev. X5 (2015) 041025 [1506.05111].

[56] R. A. Davison, W. Fu, A. Georges, Y. Gu, K. Jensen and S. Sachdev, Thermoelectric transport in disordered metals without quasiparticles: The Sachdev-Ye-Kitaev models and holography, Phys. Rev. B95 (2017) 155131 [1612.00849].

[57] S. Giombi, Higher Spin - CFT Duality, in Proceedings, Theoretical Advanced Study Institute in Elementary Particle Physics: New Frontiers in Fields and Strings (TASI 2015): Boulder, CO, USA, June 1-26, 2015, pp. 137-214, 2017, 1607.02967 , DOI.

[58] L. Fei, S. Giombi and I. R. Klebanov, Critical $O(N)$ models in $6-\varepsilon$ dimensions, Phys. Rev. D90 (2014) 025018 [1404.1094].

[59] F. Kos, D. Poland and D. Simmons-Duffin, Bootstrapping the $O(N)$ vector models, JHEP 06 (2014) $091[1307.6856]$.

[60] R. Rattazzi, V. S. Rychkov, E. Tonni and A. Vichi, Bounding scalar operator dimensions in 4D CFT, JHEP 12 (2008) 031 [0807.0004]. 
[61] D. Poland, S. Rychkov and A. Vichi, The Conformal Bootstrap: Theory, Numerical Techniques, and Applications, 1805.04405.

[62] I. R. Klebanov and A. M. Polyakov, AdS dual of the critical $O(N)$ vector model, Phys. Lett. $\mathbf{B 5 5 0}$ (2002) 213 [hep-th/0210114].

[63] M. A. Vasiliev, Consistent equation for interacting gauge fields of all spins in (3+1)-dimensions, Phys. Lett. B243 (1990) 378.

[64] S. Giombi and X. Yin, The Higher Spin/Vector Model Duality, J. Phys. A46 (2013) 214003 [1208.4036].

[65] A. Athenodorou and M. Teper, $S U(N)$ gauge theories in 2+1 dimensions: glueball spectra and $k$-string tensions, JHEP 02 (2017) 015 [1609.03873].

[66] I. R. Klebanov, World volume approach to absorption by nondilatonic branes, Nucl. Phys. B496 (1997) 231 [hep-th/9702076].

[67] J. Polchinski, Tasi lectures on D-branes, in Fields, strings and duality. Proceedings, Summer School, Theoretical Advanced Study Institute in Elementary Particle Physics, TASI'96, Boulder, USA, June 2-28, 1996.

[68] J. Polchinski, Dirichlet Branes and Ramond-Ramond charges, Phys. Rev. Lett. 75 (1995) 4724 [hep-th/9510017].

[69] H. J. Kim, L. J. Romans and P. van Nieuwenhuizen, The Mass Spectrum of Chiral N=2 D=10 Supergravity on $S^{* * 5}$, Phys. Rev. D32 (1985) 389.

[70] J. A. Minahan and K. Zarembo, The Bethe ansatz for N=4 superYang-Mills, JHEP 03 (2003) 013 [hep-th/0212208].

[71] N. Beisert et al., Review of AdS/CFT Integrability: An Overview, Lett. Math. Phys. 99 (2012) 3 [1012.3982].

[72] F. Fiamberti, A. Santambrogio, C. Sieg and D. Zanon, Wrapping at four loops in N=4 SYM, Phys. Lett. B666 (2008) 100 [0 712 . 3522].

[73] Z. Bajnok and R. A. Janik, Four-loop perturbative Konishi from strings and finite size effects for multiparticle states, Nucl. Phys. B807 (2009) 625 [0807.0399].

[74] N. Gromov, V. Kazakov and P. Vieira, Exact Spectrum of Anomalous Dimensions of Planar N=4 Supersymmetric Yang-Mills Theory, Phys. Rev. Lett. 103 (2009) 131601 [0901. 3753].

[75] N. Gromov, V. Kazakov, S. Leurent and D. Volin, Quantum Spectral Curve for Planar $\mathscr{N}=4$ Super-Yang-Mills Theory, Phys. Rev. Lett. 112 (2014) 011602 [1305.1939].

[76] N. Gromov, V. Kazakov and P. Vieira, Exact Spectrum of Planar $\mathscr{N}=4$ Supersymmetric Yang-Mills Theory: Konishi Dimension at Any Coupling, Phys. Rev. Lett. 104 (2010) 211601 [0 906.4240 ].

[77] N. Gromov and S. Valatka, Deeper Look into Short Strings, JHEP 03 (2012) 058 [1109. 6305 ].

[78] N. Gromov, F. Levkovich-Maslyuk, G. Sizov and S. Valatka, Quantum spectral curve at work: from small spin to strong coupling in $\mathscr{N}=4$ SYM, JHEP 07 (2014) 156 [1402.0871].

[79] A. Hegedus and J. Konczer, Strong coupling results in the $A d S_{5} / C F T_{4}$ correspondence from the numerical solution of the quantum spectral curve, JHEP 08 (2016) 061 [1604.0234 6].

[80] R. Roiban and A. A. Tseytlin, Semiclassical string computation of strong-coupling corrections to dimensions of operators in Konishi multiplet, Nucl. Phys. B848 (2011) 251 [1102 . 1209 ]. 
[81] M. Beccaria, S. Giombi, G. Macorini, R. Roiban and A. A. Tseytlin, 'Short' spinning strings and structure of quantum Ad $S_{5} \times S^{5}$ spectrum, Phys. Rev. D86 (2012) 066006 [1203. 5710 ].

[82] J. Ambjorn, B. Durhuus and J. Frohlich, Diseases of Triangulated Random Surface Models, and Possible Cures, Nucl. Phys. B257 (1985) 433.

[83] S. R. Das, A. Dhar, A. M. Sengupta and S. R. Wadia, New Critical Behavior in $d=0$ Large N Matrix Models, Mod. Phys. Lett. A5 (1990) 1041.

[84] I. R. Klebanov and A. Hashimoto, Nonperturbative solution of matrix models modified by trace squared terms, Nucl. Phys. B434 (1995) 264 [hep-th/9409064].

[85] I. R. Klebanov, J. M. Maldacena and N. Seiberg, Unitary and complex matrix models as 1-d type 0 strings, Commun. Math. Phys. 252 (2004) 275 [hep-th/ 0309168 ].

[86] S. Dalley, C. V. Johnson and T. R. Morris, Multicritical complex matrix models and nonperturbative 2-D quantum gravity, Nucl. Phys. B368 (1992) 625.

[87] F. Ferrari, The Large D Limit of Planar Diagrams, 1701.01171.

[88] F. Ferrari, V. Rivasseau and G. Valette, A New Large N Expansion for General Matrix-Tensor Models, 1709.07366.

[89] S. Giombi, I. R. Klebanov and G. Tarnopolsky, Bosonic tensor models at large $N$ and small $\varepsilon$, Phys. Rev. D96 (2017) 106014 [1707.03866].

[90] I. R. Klebanov and G. Tarnopolsky, On Large N Limit of Symmetric Traceless Tensor Models, 1706.00839.

[91] D. Benedetti, S. Carrozza, R. Gurau and M. Kolanowski, The $1 / N$ expansion of the symmetric traceless and the antisymmetric tensor models in rank three, 1712.00249.

[92] S. Carrozza, Large $N$ limit of irreducible tensor models: $O(N)$ rank-3 tensors with mixed permutation symmetry, JHEP 06 (2018) 039 [1803.024 96].

[93] P. Narayan and J. Yoon, SYK-like Tensor Models on the Lattice, JHEP 08 (2017) 083 [1705.01554].

[94] S. S. Gubser, C. Jepsen, Z. Ji and B. Trundy, Higher melonic theories, 1806.04800.

[95] K. Pakrouski, I. R. Klebanov, F. Popov and G. Tarnopolsky, Spectrum of Majorana Quantum Mechanics with $O(4)^{3}$ Symmetry, 1808.07455.

[96] I. R. Klebanov, A. Milekhin, F. Popov and G. Tarnopolsky, Spectra of eigenstates in fermionic tensor quantum mechanics, Phys. Rev. D97 (2018) 106023 [1802.10263].

[97] J. Polchinski and V. Rosenhaus, The Spectrum in the Sachdev-Ye-Kitaev Model, JHEP 04 (2016) 001 [1601.06768].

[98] J. Maldacena and D. Stanford, Comments on the Sachdev-Ye-Kitaev model, Phys. Rev. D94 (2016) 106002 [1604.07818].

[99] D. J. Gross and V. Rosenhaus, A Generalization of Sachdev-Ye-Kitaev, JHEP 02 (2017) 093 [1610.01569].

[100] A. M. Garcia-Garcia and J. J. M. Verbaarschot, Spectral and thermodynamic properties of the Sachdev-Ye-Kitaev model, Phys. Rev. D94 (2016) 126010 [1610.03816].

[101] J. S. Cotler, G. Gur-Ari, M. Hanada, J. Polchinski, P. Saad, S. H. Shenker et al., Black Holes and Random Matrices, JHEP 05 (2017) 118 [1611.04650]. 
[102] G. Gur-Ari, R. Mahajan and A. Vaezi, Does the SYK model have a spin glass phase?, 1806.10145.

[103] D. Benedetti and R. Gurau, 2PI effective action for the SYK model and tensor field theories, JHEP 05 (2018) $156[1802.05500]$.

[104] S. Choudhury, A. Dey, I. Halder, L. Janagal, S. Minwalla and R. Poojary, Notes on melonic $O(N)^{q-1}$ tensor models, JHEP 06 (2018) 094 [1707 . 09352].

[105] C. Krishnan, S. Sanyal and P. N. Bala Subramanian, Quantum Chaos and Holographic Tensor Models, JHEP 03 (2017) 056 [1612.06330].

[106] C. Krishnan and K. V. P. Kumar, Towards a Finite-N Hologram, JHEP 10 (2017) 099 [1706.05364].

[107] C. Krishnan and K. V. Pavan Kumar, Exact Solution of a Strongly Coupled Gauge Theory in 0+1 Dimensions, Phys. Rev. Lett. 120 (2018) 201603 [1802 . 02502].

[108] K. Symanzik, On Calculations in conformal invariant field theories, Lett. Nuovo Cim. 3 (1972) 734.

[109] A. Almheiri and J. Polchinski, Models of AdS 2 backreaction and holography, JHEP 11 (2015) 014 [1402.6334].

[110] A. Jevicki, K. Suzuki and J. Yoon, Bi-Local Holography in the SYK Model, JHEP 07 (2016) 007 [1603.06246].

[111] J. Maldacena, D. Stanford and Z. Yang, Conformal symmetry and its breaking in two dimensional Nearly Anti-de-Sitter space, PTEP 2016 (2016) 12C104 [1606. 01857 ].

[112] J. Engelsoy, T. G. Mertens and H. Verlinde, An investigation of $A d S_{2}$ backreaction and holography, JHEP 07 (2016) 139 [1606.03438].

[113] K. Jensen, Chaos in AdS 2 Holography, Phys. Rev. Lett. 117 (2016) 111601 [1605. 06098 ].

[114] J. Ben Geloun and S. Ramgoolam, Counting Tensor Model Observables and Branched Covers of the 2-Sphere, 1307.6490.

[115] M. Beccaria and A. A. Tseytlin, Partition function of free conformal fields in 3-plet representation, JHEP 05 (2017) 053 [1703.04460].

[116] H. Itoyama, A. Mironov and A. Morozov, Ward identities and combinatorics of rainbow tensor models, JHEP 06 (2017) 115 [1704.08648].

[117] A. Mironov and A. Morozov, Correlators in tensor models from character calculus, 1706.03667.

[118] P. Diaz and S.-J. Rey, Orthogonal Bases of Invariants in Tensor Models, 1706.02667.

[119] R. de Mello Koch, D. Gossman and L. Tribelhorn, Gauge Invariants, Correlators and Holography in Bosonic and Fermionic Tensor Models, 1707.01455.

[120] S. Dartois, V. Rivasseau and A. Tanasa, The 1/N expansion of multi-orientable random tensor models, Annales Henri Poincare 15 (2014) 965 [1301.1535].

[121] W. Fu, D. Gaiotto, J. Maldacena and S. Sachdev, Supersymmetric Sachdev-Ye-Kitaev models, Phys. Rev. D95 (2017) 026009 [1610.08917].

[122] K. Bulycheva, A note on the SYK model with complex fermions, JHEP 12 (2017) 069 [1706.07411].

[123] J. Yoon, SYK Models and SYK-like Tensor Models with Global Symmetry, JHEP 10 (2017) 183 [1707.01740]. 
[124] C. Peng, M. Spradlin and A. Volovich, Correlators in the $\mathscr{N}=2$ Supersymmetric SYK Model, JHEP 10 (2017) 202 [1706.06078].

[125] P. Chaturvedi, Y. Gu, W. Song and B. Yu, A note on the complex SYK model and warped CFTs, 1808.08062 .

[126] J. Kim, Large N Tensor and SYK Models, Princeton University Senior Thesis, 2018 , .

[127] R. Gurau, The complete 1/N expansion of a SYK-like tensor model, 1611.04032.

[128] P. Breitenlohner and D. Z. Freedman, Stability in Gauged Extended Supergravity, Annals Phys. 144 (1982) 249.

[129] A. Dymarsky, I. R. Klebanov and R. Roiban, Perturbative search for fixed lines in large $N$ gauge theories, JHEP 08 (2005) 011 [hep-th/ 050509 9].

[130] E. Pomoni and L. Rastelli, Large N Field Theory and AdS Tachyons, JHEP 04 (2009) 020 [0805.2261].

[131] D. Grabner, N. Gromov, V. Kazakov and G. Korchemsky, Strongly $\gamma$-Deformed $\mathscr{N}=4$ Supersymmetric Yang-Mills Theory as an Integrable Conformal Field Theory, Phys. Rev. Lett. 120 (2018) 111601 [1711.04786].

[132] S. Prakash and R. Sinha, A Complex Fermionic Tensor Model in d Dimensions, JHEP 02 (2018) 086 [1710.09357].

[133] I. R. Klebanov and E. Witten, AdS / CFT correspondence and symmetry breaking, Nucl. Phys. B556 (1999) 89 [hep-th/9905104].

[134] J. Liu, E. Perlmutter, V. Rosenhaus and D. Simmons-Duffin, d-dimensional SYK, AdS Loops, and $6 j$ Symbols, 1808.00612 .

[135] J. Murugan, D. Stanford and E. Witten, More on Supersymmetric and 2d Analogs of the SYK Model, JHEP 08 (2017) 146 [1706.05362].

[136] C.-M. Chang, S. Colin-Ellerin and M. Rangamani, On Melonic Supertensor Models, 1806.09903. 